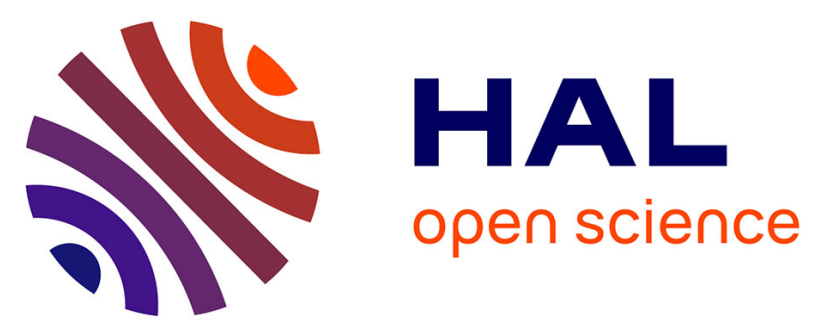

\title{
The mineral weathering ability of Collimonas pratensis PMB3(1) involves a Malleobactin-mediated iron acquisition system
}

Laura Picard, Cédric Paris, Tiphaine Dhalleine, Emmanuelle Morin, Philippe Oger, Marie-Pierre M.-P. Turpault, Stéphane Uroz

\section{To cite this version:}

Laura Picard, Cédric Paris, Tiphaine Dhalleine, Emmanuelle Morin, Philippe Oger, et al.. The mineral weathering ability of Collimonas pratensis PMB3(1) involves a Malleobactin-mediated iron acquisition system. Environmental Microbiology, 2022, 24 (2), pp. 784-802. 10.1111/1462-2920.15508 . hal03194986

\section{HAL Id: hal-03194986 \\ https://hal.science/hal-03194986}

Submitted on 9 Apr 2021

HAL is a multi-disciplinary open access archive for the deposit and dissemination of scientific research documents, whether they are published or not. The documents may come from teaching and research institutions in France or abroad, or from public or private research centers.
L'archive ouverte pluridisciplinaire HAL, est destinée au dépôt et à la diffusion de documents scientifiques de niveau recherche, publiés ou non, émanant des établissements d'enseignement et de recherche français ou étrangers, des laboratoires publics ou privés. 
1 TITLE: The mineral weathering ability of Collimonas pratensis PMB3(1) involves a

2 Malleobactin-mediated iron acquisition system

4 Running title: Mineral weathering role of Malleobactin

6 AUTHORS: Laura Picard ${ }^{1,2}$, Cédric Paris 3,4 , Tiphaine Dhalleine ${ }^{1}$, Emmanuelle Morin1,

7 Philippe Oger ${ }^{5}$, Marie-Pierre Turpault², Stéphane Uroz ${ }^{1,2}$

81 Université de Lorraine, INRAE, UMR1136 « Interactions Arbres-Microorganismes »,

9 F-54280 Champenoux, France

102 INRAE, UR1138 « Biogéochimie des Ecosystèmes Forestiers 》, F-54280

11 Champenoux, France

123 Université de Lorraine, EA 4367 "Laboratoire d'Ingénierie des Biomolécules »,

13 Ecole Nationale Supérieure d'Agronomie et des Industries Alimentaires (ENSAIA), F-

1454505 Vandœuvre-lès-Nancy, France

$15{ }^{4}$ Plateau d'Analyse Structurale et Métabolomique (PASM) - SF4242 EFABA, F-

1654505 Vandœuvre-lès-Nancy, France 
175 Univ Lyon, INSA de Lyon, CNRS UMR 5240 « Microbiologie, Adaptation et

18 Pathogénie », F-69621, Villeurbanne France

19

20 * Corresponding author: Mailing address:

21 Université de Lorraine, INRAE, UMR 1136 "Interactions Arbres Microorganismes",

2254280 Champenoux, France. Phone: +33 (0)3 833940 81, Fax: +33 (0)3 833940

23 69. E-mail: stephane.uroz@inrae.fr. 


\section{SUMMARY}

25 Mineral weathering (MW) by microorganisms is considered to occur through a

26 succession of molecular mechanisms based on acidification and chelation. While the

27 role of acidification is established, the role of siderophores is difficult to disentangle

28 from the effect of the acidification. We took advantage of the ability of strain

29 Collimonas pratensis PMB3(1) to weather minerals but not to produce organic acids

30 depending on the corbon source to adress the role of siderophores in MW. We

31 identified a single non-ribosomal peptide synthetase (NRPS) responsible for

32 siderophore biosynthesis in the PMB3(1) genome. By combining iron-chelating

33 assays, targeted mutagenesis and chemical analyses (HPLC and LC-ESI-HRMS),

34 we identified the siderophore produced by strain PMB3(1) as malleobactin $\mathrm{X}$ and how

35 its production depends on the concentration of available iron. Comparison with the

36 genome sequences of other collimonads evidenced that malleobactin production

37 seems to be a relatively conserved functional trait, though some collimonads

38 harbored other siderophore synthesis systems. We also revealed by comparing the

39 wild-type strain and its mutant impaired in the production of malleobactin that the

40 ability to produce this siderophore is essential to allow the dissolution of hematite 3 
41 under non-acidifying conditions. This study represents the first characterization of the

42 siderophore produced by collimonads and its role in mineral weathering.

44 Key words: Collimonas pratensis PMB3(1); mineral weathering; siderophore;

45 malleobactine; hematite

46

47

48

49

50

51 Originality-Significance Statement

52 Nutrient access is an essential process in nutrient-poor and non-amended

53 ecosystems. To deal with such conditions, plants have developed particular strategies to

54 adapt, noticeably through the selection in their rhizosphere of functional communities

55 effective at mobilizing the nutrients. Among them, bacteria have been shown to be

56 effective at mineral weathering (MW) and at promoting plant growth. Acidification and

57 chelation are considered as the main mechanisms used by MW bacteria. However, 
58 our understanding of the molecular mechanisms and genes involved remains limited.

59 While the role of acidification is established, the role of siderophores is difficult to

60 disentangle from the effect of the acidification. In strain PMB3(1) of Collimonas

61 pratensis, the production of organic acid by the central metabolism is dependent on

62 the carbon source used for growth. Hence, it is one of the few bacterial models in

63 which it is possible to decipher mineral weathering molecular mechanisms that are

64 organic acid-independent. Strain PMB3(1) of Collimonas pratensis is a very effective

65 MW bacterium, particularly adapted to oligotrophic environments and effective at

66 promoting plant growth and at protecting plants against fungal pathogens. Mineral

67 weathering has been proposed to be a functional trait of this genus, which molecular

68 mechanisms have not yet been elucidated. The novel results obtained in this study

69 through the combination of bioinformatics, chemical and phylogenetic analyses

70 allowed us to identify and to characterized the siderophore produced by this model

71 strain and to demonstrate the role of this iron acquisition system in mineral

72 weathering. This study represents the first experimental demonstration of the

73 siderophore produced by Collimonas (i.e., malleobactin). Our analyses allowed us to

74 evidence the conservation of this iron acquisition system among collimonads and to 5 
75 improve the siderophore prediction tools, especially to better discriminate ornibactin 76 and malleobactin. Our findings offer relevant informations for different fields of

77 research such as environmental genomics and chemistry, and soil sciences.

78 


\section{INTRODUCTION}

80 Iron is an essential micronutrient for living organisms. It is involved in key cellular

81 processes such as DNA synthesis, respiration, photosynthesis or metabolism (Rout and Sahoo,

82 2015). Due to its oxidoreduction potential iron is used by several enzymes as energy source

83 (i.e. cytochrome subunit of enzymes or cofactors). Although this element is abundant in the

84 Earth crust, its bioavailability is strongly reduced in aerobic soils ranging from neutral to

85 basic $\mathrm{pH}$, while in acidic soils such as in temperate forests, its bioavailability is higher.

86 However, it tends to decrease from the topsoil horizon to the deeper horizons due to the

87 precipitation of iron as iron oxi-hydroxides and its adsorption on different substrates such

88 as organic matter and primary minerals. In soils, the main sources of iron are the primary

89 minerals (i.e., granite and other Fe-carrying minerals and rocks), the secondary

90 minerals such as iron oxi-hydroxides (i.e., hematite, goethite) and the adsorbed

91 forms. However, most of those minerals dissolve at much slower rates than those required to

92 support plant and microbial growth (Lindsay, 1995). Consequently, iron access is subject to

93 important competitions between living organisms (Butaitè et al., 2017).

94 The ability to mobilize iron from the different soil compartments (i.e., minerals, rocks,

95 organic matter) has been reported for several plants, fungi, and bacteria. Noticeably, effective

96 iron mobilizing bacteria have been evidenced in the rhizosphere of various plants and in

97 different soils (Uroz et al., 2009a). The presence of such bacteria and their relative enrichment

98 in the rhizosphere compared to the surrounding bulk soil suggest that these functional

99 communities are recruited in the root environment to make iron bioavailable for plant

100 nutrition. Among the effective iron mobilizing bacteria encountered in nutrient-poor forest 
101 soils, bacteria belonging to the genus Collimonas have been described for their effectiveness

102 in mobilizing iron (Uroz et al., 2009b, 2007; Leveau et al., 2010). Although, collimonads

103 are considered as members of the rare biosphere in the soil (Leveau et al., 2010),

104 they can be the dominant taxa in specific habitats such as in the mineralosphere

105 (Uroz et al., 2015) or in the mycorrhizosphere (Lepleux et al., 2012; Uroz et al.,

106 2012). Representatives of this genus have been isolated from various nutrient-poor

107 environments (i.e., grass land, forests soil; (Uroz et al., 2009b, 2014; Hoppener-

108 Ogawa et al., 2008) and often in the close vicinity of fungi (De Boer, 2004; Uroz et al.,

109 2012). Some Collimonas species have been used as plant growth promoting agent

110 (e.g. tomatoes) to protect them against fungal pathogens (Kamilova et al., 2007;

111 Senechkin et al., 2013; Doan et al., 2020) and to promote the growth of Pinus

112 sylvestris seedlings (Koele et al., 2009). All these characteristics make this genus an

113 interesting plant growth-promoting (PGP) candidate.

114 The functional screening of Collimonas collections revealed that all the collimonads were 115 especially effective at weathering minerals compared to other genera. Among them, 116 Collimonas pratensis strain PMB3(1) appeared as the most effective (Uroz et al., 2009b; 117 Picard et al., 2020). This strain is able to solubilize inorganic phosphorous, mobilize iron and 118 dissolve minerals. At the molecular level, this high ability to weather mineral is explained by 119 the ability of the collimonads to produce protons via the direct oxidative pathway (Picard et 
al., 2021). Strain PMB3(1) is capable of strongly acidifying its environment when glucose is

121 used as sole carbon source and to produce high concentration of gluconic acid (Uroz et al.,

122 2009b; Picard et al., 2021). In that respect, the glucose/methanol/choline (GMC)

123 oxidoreductase was identified as responsible for this acidification process in strain PMB3(1)

124 (Picard et al., 2021). Other molecular mechanisms such as complexolysis may also play a role

125 in the dissolution of minerals (Uroz et al., 2009a; Dong, 2010). Indeed, most of the bacteria

126 recruited in the rhizosphere are capable of producing siderophores. To date, different types of

127 siderophores have been reported and new chelating molecules are continuously discovered

128 (Hernandez et al., 2004; Mathew et al., 2014). Siderophores can impact directly (i.e., surface

129 dissolution) or indirectly (i.e., modification of the solution equilibrium) the mineral

130 weathering process, especially when carbon sources other than glucose are available (i.e.,

131 mannitol, trehalose) (Holmén and Casey, 1996; Kraemer, 2004; Parrello et al., 2016; Perez et 132 al., 2019).

133 In absence of acidification, the dissolution of minerals may occur through the action of 134 siderophores (Shirvani and Nourbakhsh, 2010), but no experimental study was done on 135 collimonads to functionally characterize the gene clusters involved and the type of 136 siderophore produced. The ability to chelate iron was evidenced for several Collimonas 137 strains (Uroz et al., 2009b; Ballhausen et al., 2016). More recently, bioinformatics analyses

138 identified genes encoding a potential Non-Ribosomal Peptide Synthetase (NRPS) in the

139 genomes of collimonads (i.e., Ter6, Ter91, Ter331, Ter10, Ter282 and Ter291) and predicted 140 the production of ornibactin (Song et al., 2015). In this context, the goals of this study were i)

141 to identify and chemically characterize the siderophore produced by the model Collimonas 142 pratensis strain PMB3(1), ii) to investigate how conserved the gene sequences were among 143 collimonads and related genera and iii) to determine the role of the siderophore in mineral 144 weathering. To do this, we combined bioinformatics, genomics, genetics, geochemistry and 9 
145 functional bioassays to decipher the genes conferring the chelating and mineral weathering

146 abilities to strain PMB3(1) and other collimonads. The use of high-performance liquid

147 chromatography (HPLC) coupled to high-resolution and tandem mass spectrometry

148 (HRMS and MS/MS) allowed us to identify the siderophore produced as malleobactin X.

150 RESULTS

151 Identification of a genomic region involved in siderophore production

152 The CAS plate assay revealed that the model Collimonas pratensis strain

153 PMB3(1) produced chelating molecules as shown by the development of a yellow

154 halo around the colony (Fig. 1A). To identify the genomic region potentially involved

155 in the production of chelators in strain PMB3(1), an antiSMASH search was

156 performed on the genome sequence. Such analysis identified 11 regions encoding

157 putative polyketide synthases (PKS; n=2) or Non-Ribosomal Peptide Synthetases

158 (NRPS; $n=9$ ) with prediction or not of the final product (Table S2). Among the

159 potential NRPS, only one was predicted as encoding a siderophore. This region large

160 of $27.7 \mathrm{~kb}$ is characterized by 13 genes (Fig. 2; Table 2). Six genes are involved in

161 siderophore biogenesis. Among them the larger genes $m b a A$ and $m b a B$ encode 
162 Non-ribosomal peptide synthetase (NRPS) composed of modules which including

163 domains (Condensation, Adenylation, Thiolation). In addition, the region is

164 characterized by 6 genes involved in siderophore transport and utilization and by one

165 gene encoding a sigma factor.

166

167 Prediction of the siderophore structure

168 To predict the structure of the siderophore, the two NRPS encoding genes ( $m b a A$

169 NKI69295.1 and $m b a B$ NKI69296.1) were submitted to NRPS prediction using three

170 different tools. All predictions identified four biosynthetic modules, three were

171 encoded by the $m b a A$ gene and the last one by the mbaB gene. Modules are

172 composed of different domains: adenylation $(A)$, thiolation $(T)$, condensation $(C)$ and

173 epimerization (E) following the schema (AT)-(CATE)-(CAT)-(CATC) for modules 1-2-

174 3-4 respectively. The NRPS prediction tools also proposed the amino acid

175 composition of the siderophore based on the Stachelhaus code (Stachelhaus et al.,

176 1999). For modules 2 and 3, the three NRPS prediction tools proposed the same

177 amino acids. Module 2 corresponds to a surfactin synthetase B ( $75 \%$ identity) that

178 recruits an aspartate (Asp). Module 3 has $100 \%$ identity with a pyoverdin synthetase 11 
179 that recruits a serine (Ser). No consensus was obtained for modules 1 and 4. Indeed,

180 the signature sequence (DVETLGGISK or DVETLGGI) identified for module 1

181 indicated the recruitment of beta-hydroxy-tyrosine according to 'NRPS predictor 2' or

182 an ornithine according to 'NP.searcher'. For the amino acid recruited by the module

183 4, the signature sequence was DGEYTGGITK or DGEYTGGI. Such sequence is

184 predicted to recruit a leucine according to 'NRPS predictor 2' and 'NP.searcher', or

185 an ornithine according to 'PKS/NRPS analysis'. Altogether, our bioinformatics

186 analyses predicted a siderophore composed of four amino acids, the second being a

187 serine and the third an aspartate (X-Ser-Asp-X).

189 Involvement of the mbaA gene in the production of siderophore

190 To demonstrate the involvement of this NRPS encoding region in siderophore

191 synthesis, a mbaA mutant named $\triangle$ NRPS was constructed and tested using different

192 bioassays (i.e., siderophore production, growth assay).

193 Regarding the ability to chelate iron, the WT strain of PMB3(1) was able to

194 mobilize iron in the CAS plate assay after 3 days. The yellow halo formed around the

195 colonies (WT colony diameter: $1.0 \pm 0.1 \mathrm{~cm}$ ) was about $1.5 \pm 0.1 \mathrm{~cm}$, while no halo 
196 was observed for the $\triangle$ NRPS mutant strain ( $\triangle$ NRPS colony diameter: $0.6 \pm 0.1 \mathrm{~cm}$ )

197 (Fig. 1A). The production of chelating agent by the WT strain was also confirmed in

198 liquid CAS assay where the absorbance in absence of iron in the culture medium is

199 significantly decreased $(\mathrm{OD}$ at $655 \mathrm{~nm}=0.053 \pm 0.001)$ compared to the control.

200 Noticeably, for the WT strain, a siderophore activity was observed for iron

201 concentration ranging from 0 to $0.7 \mathrm{mg} / \mathrm{l}$, while the production of chelator is inhibited

202 for greater values (iron concentration of $0.8 \mathrm{mg} / \mathrm{l}$ : OD at $655 \mathrm{~nm}=0.372 \pm 0.019$;

$203 \mathrm{P}<0.05)$ (Fig. 1B). Whatever the iron concentration, the $\triangle$ NRPS mutant did not

204 present a chelating activity (OD at $655 \mathrm{~nm}=0.458 \pm 0.005)$ (Fig. 1B).

206 Impact of the mbaA mutation on growth

207 To determine how the concentration of iron and the mutation of the $m b a A$ gene

208 ( $\triangle$ NRPS mutant) affect the growth of strain PMB3(1), were performed tests in

209 medium containing increasing concentrations of iron (i.e. $0.1 ; 0.2 ; 0.3 ; 0.5 ; 1.0 \mathrm{mg} / \mathrm{l}$ ).

210 In this way, we demonstrated that the growth rate increased with increasing

211 concentrations of iron and that strain PMB3(1) and its $\triangle$ NRPS mutant presented the

212 same growth rate $(P>0.05)$ (Fig. S1). With $1 \mathrm{mg} / \mathrm{l}$ of iron, the growth rates of the 13 
$213 \Delta$ NRPS mutant and WT strains without EDTA were ca $0.06 \pm 0.005 \mathrm{~h}^{-1}$. However, in

214 presence of EDTA (10 mM), no growth was observed for the $\triangle$ NRPS mutant while

215 the growth of the WT strain was maintained, but its growth rate decreased to $0.01 \pm$

$2160.005 \mathrm{~h}^{-1}$ (Fig. 1C).

218 Purification of the siderophore and chemical characterization

219 To determine the structure of the synthetized siderophore, the supernatants of 3-

220 days old cultures in ABm devoid of iron of the WT strain (containing the siderophore),

221 the $\triangle$ NRPS mutant strain and the medium were analysed by HPLC and LC-ESI-MS

222 (or MS/MS).

223 The HPLC chromatograms of the WT, $\triangle$ NRPS mutant and medium supernatants

224 are presented in figure $3 \mathrm{~A}$. Such analysis evidenced a major peak with a retention

225 time (RT) of 1.2 min only present for the WT strain and absent from the two other

226 conditions. To investigate the potential presence of a siderophore activity associated

227 to this peak, the collected HPLC fractions were tested with the liquid CAS assay.

228 Both HPLC chromatogram and siderophore activity were overlaid and are presented

229 in figure 3B. Noticeably, the CAS assay revealed a strong reduction of the 
230 absorbance $\left(\mathrm{OD}_{655 \mathrm{~nm}}=0.055\right)$ for the fractions corresponding to the peak present at

$231 \mathrm{RT}=1.2 \mathrm{~min}$, matching with the yellow color observed with the CAS assay. This

232 confirmed the presence of a chelating activity in the first peak. In addition, two other

233 peaks were detected for the WT strain at 2.2 (peak 2) and 3.8 (peak 3) min RT (Fig.

$2343 \mathrm{~A})$. The liquid CAS assay revealed a decrease of absorbance $\left(\mathrm{OD}_{655 \mathrm{~nm}}=0.110\right.$ and

2350.103 for peak 2 and 3, respectively; Fig. 3B) after $1 \mathrm{~h}$ incubation, suggesting the

236 presence of a chelating activity in the two peaks.

237 A deeper analysis was done by liquid chromatography coupled to positive ion-

238 mode electrospray mass spectrometry (LC-ESI+-HRMS) on the first peak collected to

239 perform the chemical characterization on a fraction which chelating activity was

240 demonstrated. High resolution MS analysis revealed that the siderophore compound

241 (S) has an exact mass of $M_{S}=622.3069$ (monoprotonated ion seen at

$242 \mathrm{~m} / \mathrm{z}=623.3069$ ) (Fig. $4 \mathrm{~A}$ ). This value is strictly similar to the mass of the

243 malleobactin $X$ (Fig. 4E). To confirm the malleobactin $X$ structure, the siderophore

244 was further analyzed by tandem mass spectrometry (MS/MS) and the major product

245 ions (Fig. 4C) compared with theoretical fragments (Fig. 4D). Product ion at $\mathrm{m} / \mathrm{z}=$

246535.4 (Fig. 4C; b4) was attributed to the initial loss of C-terminal putrescine from the 
247 precursor ion and that at $\mathrm{m} / \mathrm{z}=517.4$ to additional dehydration $\left(\mathrm{H}_{2} \mathrm{O}\right.$ loss from $\mathrm{m} / \mathrm{z}=$

248 535.4). Product ion at $\mathrm{m} / \mathrm{z}=465.3$ (also seen in its dehydrated form at $\mathrm{m} / \mathrm{z}=447.4$ )

249 (Fig. 4C; y1) was attributed to the initial loss of $\mathrm{N}$-terminal ornithine from the

250 precursor ion. The breaking of the peptide bond between serine and ornithine gave

251 rise to a product ion at $\mathrm{m} / \mathrm{z}=377.2$ (also seen in its dehydrated form at $\mathrm{m} / \mathrm{z}=359.2$ )

252 (Fig. 4C; b3) and another product ion at $\mathrm{m} / \mathrm{z}=247.3$ (Fig. 4C; y3). The breaking

253 between aspartate and serine gave rise to a product ion at $\mathrm{m} / \mathrm{z}=334.3$ (Fig. $4 \mathrm{C} ; \mathrm{y} 2$ ).

254 An additional LC-ESI+-HRMS analysis was performed on the same fraction

255 (collected at RT $=1.2 \mathrm{~min}$ ), but previously incubated with iron (see details in

256 Ferrisiderophore preparation section). As presented on Figure 4B, formation of the

257 expected ferrisiderophore (FS) was confirmed through the clear observation of the

258 ion at $\mathrm{m} / \mathrm{z}=676.2191\left(\mathrm{M}_{\mathrm{FS}}(\exp )=675.2191\right)$. This experimental value is in full

259 agreement with the complexation between iron (55.935 u for major isotope $\left.{ }^{56} \mathrm{Fe}\right)$ and

260 the triply deprotonated siderophore $S(622.3069-3 \times 1.008=619.2829 u)$, leading to

261 a theoretical mass $\mathrm{MFS}_{\text {(theo) }}=675.2179(55.935+619.2829)$. The presence of iron in

262 the structure was also validated by the detection of the characteristic isotopic pattern

263 detailed in Figure 4B, showing clearly the contributions of ${ }^{54} \mathrm{Fe}$ and ${ }^{56} \mathrm{Fe}$ isotopes 
$264(\mathrm{~m} / \mathrm{z}=674.2246$ and 676.2191 , respectively $)$. In addition, the relative intensities of 265 the ions at $\mathrm{m} / \mathrm{z}=677.2213(28.8 \%)$ and at $\mathrm{m} / \mathrm{z}=678.2236(4.4 \%)$ were found to be 266 in perfect accordance with the predicted isotopic distribution for malleobactin (Manura

267 and Manura, 2015). The MS/MS analysis conducted on the ferrisiderophore FS 268 (fragmentation of the ion at $\mathrm{m} / \mathrm{z}=676.2$ ) revealed a product ion at $\mathrm{m} / \mathrm{z}=$ 269588.3 explained by the loss of C-terminal putrescine (dehydrated form also seen at $270 \mathrm{~m} / \mathrm{z}=570.2)($ Fig. S2). A second intense ion at $\mathrm{m} / \mathrm{z}=606.2802$ was also observed

271 on Figure $4 \mathrm{~B}$, in addition to that attributed to the ferrisiderophore $\mathrm{FS}(\mathrm{m} / \mathrm{z}=$ 272 676.2191).

273 The experimental masses found for the siderophore S (without incubation with

274 iron) and for the ferrisiderophore FS (after incubation of the siderophore with iron)

275 agree with the results previously obtained on Burkholderia xenovorans strain LB400

276 (Vargas-Straube et al., 2016), allowing us to clearly identify the malleobactin $\mathrm{X}$ as the 277 siderophore produced by strain PMB3(1). 
280 Our phylogenetic analysis revealed a good differentiation of the NRPS proteins according 281 to the chemical structure of the siderophores (Fig. 5; green, ornibactin; red,

282 malleobactin), the taxonomic belonging and the MbaA protein sequence. Beside the

283 serobactin-related cluster, two clusters can be identified. The cluster 1 is supported

284 by NRPS sequences (i.e., Orbl homologues) assigned to Burkholderia cepacia, $B$.

285 cenocepacia or B. vietnamiensis and is predicted to produce ornibactin. Noticeably,

286 the production of ornibactin was experimentally demonstrated for three strains (B.

287 contaminans MS14 (Deng et al., 2017), B. cenocepacia K56-2 (Darling et al., 1998), B.

288 cepacia ATCC25416 (Meyer et al., 1995)) of this cluster. The second cluster is composed

289 of NRPS sequences (i.e., MbaA homologues) assigned to Burkholderia

290 pseudomallei, B. mallei, B. thailandensis and Collimonas and predicted to produce

291 ornibactin or malleobactin. In this cluster, the production of malleobactin was

292 experimentally demonstrated for three strains (B. pseudmallei K96243 (Alice et al., 2006), B.

293 thailandensis (Franke et al., 2013), B. xenovorans LB400 (Vargas-Straube et al., 2016)),

294 while the production of ornibactin was only predicted. The strain PMB3(1) represents the

295 fourth strains in this cluster which malleobactin production was experimentally demonstrated

296 and the first collimonads. The strong proximity between the MbaA protein of the

297 collimonads, the ability of strain PMB3(1) to produce malleobactin and the conserved ability

298 to mobilize iron in collimonads suggest that all the collimonads considered synthetize

299 malleobactin and not ornibactin as previously predicted (Fig. 5, green dotted circle; (Song et 300 al., 2015). Noticeably, the most distantly related sequence (38.25\% of identity) to MbaA of 
301 strain PMB3(1) is the NRPS protein responsible of the biosynthesis of serobactin in

302 Herbaspirillum seropedicae that is however the closest genus of Collimonas according to

303 taxonomy (Rosconi et al., 2013).

304

305 Siderophore involvement in hematite weathering

306 To evaluate the potential involvement of the siderophore produced by Collimonas

307 strain PMB3(1) on mineral weathering, a hematite dissolution experiment was

308 performed (Fig. 6). To exclude an acidification effect, the experiment was done with

309 mannitol as sole carbon source, which was previously shown not to lead to the

310 acidification of the medium during growth (Uroz et al., 2009b). After 7-days of

311 incubation, the iron released in solution from hematite as well as the $\mathrm{pH}$ were

312 measured, permitting to monitor the mineral weathering potential of the different

313 strains tested. In this experiment, no significant growth difference was observed

314 between the $\triangle$ NRPS mutant and the WT strains $\left(\mathrm{OD}_{595 \mathrm{~nm}}=0.8 \pm 0.1 ; \mathrm{P}<0.05\right)$,

315 though the WT strain released significantly more iron from the mineral $(0.99 \pm 0.19$

$316 \mathrm{mg} / \mathrm{l}$; Fig. 6) than the $\triangle$ NRPS mutant strain or the non-inoculated controls (i.e. $0.01 \pm$

$3170.01 \mathrm{mg} / \mathrm{l} ; \mathrm{P}<0.05)$. Noticeably, the $\mathrm{pH}$ of the medium remained stable at 6.2

318 whatever the treatment (inoculated with the WT strain, inoculated with the $\triangle$ NRPS 19 
319 mutant strain and non-inoculated controls). In this sense, the CAS assay performed

320 on the supernatant for each condition demonstrated a siderophore activity (yellow

321 colour) only for the WT strain (Fig. 6).

\section{DISCUSSION}

324 The high effectiveness of Collimonas pratensis strain PMB3(1) to weather mineral

325 led us to investigate in more details the molecular mechanisms involved. In a

326 previous study, we showed that the mineral weathering effectiveness of this strain

327 was related to the carbon substrate used (i.e., glucose, mannitol, trehalose,

328 gluconate; (Uroz et al., 2009b)). In presence of glucose this strain strongly acidified the

329 medium and effectively weathers minerals, through the production of protons and

330 gluconic acids (Picard et al., 2021). Noticeably, the strain PMB3(1) appeared also

331 capable of weathering minerals in presence of mannitol, a sugar highly produced by

332 mycorrhizal fungi and metabolized by this bacterial strain through a moderately-

333 acidifying pathway. The mineral weathering ability observed with mannitol suggests

334 the existence of alternative MW mechanisms such as iron acquisition systems. In this

335 study, we investigated the mechanisms by which this strain was able to mobilize iron 
336 from artificial (i.e., CAS) or natural iron complexes (i.e., hematite). The in silico

337 analyses revealed that the genome of strain PMB3(1) harbors the ftrABCD system

338 (siderophore-independent iron uptake system; (Mathew et al., 2014): [NKI69657.1-

339 NKI69654.1]), the bacterioferritin (iron storage protein; (Andrews, 1998): [NKI72009.1-

340 NKI72013.1]), the fecIR operon ((Braun et al., 2003): [NKI68784.1-NKI68785.1]), and an

341 incomplete hmu operon ((Nienaber et al., 2002); only hmuU [NKI72430.1] and hmuV

342 [NKI72429.1] were identified). In addition, an antiSMASH analysis identified a NRPS

343 cluster, predicted to synthetize a siderophore (i.e., ornibactin). The presence in this

344 strain of all these iron acquisition systems suggests that it is quite adapted to the nutrient-poor

345 conditions occurring in the soil where it was isolated, but may also reflect the competition for

346 iron occurring in the mycorrhizosphere with other bacteria and fungi.

348 Although, strain PMB3(1) possesses all these putative iron acquisition systems, the knock-

349 out of a single gene of the NRPS cluster fully abolished its ability to mobilize iron as seen

350 using the CAS assay. These results suggest that the other iron acquisition systems play a

351 minor role in comparison to the production of siderophores or that they are important in other

352 processes such as the mobilization of intracellular iron reserves. The NRPS region detected

353 in strain PMB3(1) presents the classical structure found in other bacterial species

354 producing NRPS-dependent siderophores with the identification of genes involved in

355 siderophore biogenesis (including the 2 NRPS genes), in siderophore transport and 
356 utilization (Fig. 2; (Esmaeel et al., 2016, 2018; Butt and Thomas, 2017)). Noticeably, the

$357 m b a A$ and $m b a B$ genes coding for siderophore production presented $36 \%$ identity

358 with genes $m b a A$ and $m b a B$ of $B$. xenovorans LB400, a strain capable of chelating

359 iron and producing malleobactin (Vargas-Straube et al., 2016). Different homologues of

360 enzymes involved in $\mathrm{N}$ - and $\mathrm{C}$-terminal modifications during siderophore production

361 were also detected. These included $\mathrm{mbaH}$ which encodes a taurine dioxygenase

362 TauD/F involved in the modifications on the $\mathrm{N}$-terminal ornithine (Franke et al., 2013),

$363 \mathrm{mbaC}$ which encodes a L-ornithine 5-monooxygenase involved in the formation of a

364 nitrogroup (Franke et al., 2013) and mbaE encoding a $\mathrm{N}(5)$-hydroxyornithine

365 transformylase PvdF-type involved in modifications of the C-terminal ornithine

366 (McMorran et al., 2001; Vargas-Straube et al., 2016). The mbal, J, L, N and D genes

367 involved in the ferrisiderophore complex recognition and transport were also

368 identified (Butt and Thomas, 2017). In addition, the enzyme in charge of the

369 reduction of the ferrisiderophore and of the release of $\mathrm{Fe}^{2+}$ and siderophore in the

370 cytoplasm was identified as the mbaK gene encoding a siderophore-iron reductase

371 FhuF (Matzanke et al., 2004). The mbaF gene responsible of the siderophore

372 expression and regulation was also identified as well as mbaF-dependent promoters 
373 with specific -35 and -10 regions (TAAA/n(17)/CGTC) (Agnoli et al., 2006; Thomas,

374 2007). Last, two genes (iorA, iorB) were identified at the end of the siderophore

375 biosynthesis region. They are predicted to encode two subunits of an aldehyde

376 dehydrogenase responsible of the formation of a carboxyl group. This function may

377 be responsible of modifications occurring after the synthesis of the siderophore.

378 However, a $\Delta$ lorB mutant presented no differences in siderophore mass $\left(\mathrm{Ms}_{\mathrm{s}}=\right.$

379622.3069 ) or in its chelating activity compared to the WT strain (data not shown). A

380 comparison with all the other available collimonads genomes revealed a good

381 conservation of the NRPS regions as well as of the iorAB genes. Indeed, the

382 complete NRPS region was found in nine of the 14 genomes and was absent from

383 the other five (Collimonas sp. OK242; Collimonas sp. OK307; Collimonas sp. OK607;

384 Collimonas sp. PA-H2 and Collimonas arenae Cal35). A more in-depth analysis of

385 the genomes of these collimonads revealed that some of them possessed genes

386 involved in the synthesis of other siderophores predicted to be myxochelin (41\% of

387 homology for strains Cal35 and PA-H2) or staphylobactin (18\% of homology for

388 strains OK242 and OK307). No siderophore region was detected for strain OK607. 
389 These results suggest that malleobactin may not be the sole siderophore produced

390 by collimonads.

392 The combination of HPLC, fraction collect and mass spectrometry allowed us to

393 identify the siderophore produced by Collimonas pratensis strain PMB3(1) as

394 malleobactin. This hydroxamate type siderophore was reported for the first time to be

395 produced by Pseudomonas pseudomallei (reclassified as Burkholderia pseudomallei)

396 hence its name (Yang et al., 1991). To date, different forms of malleobactin have been

397 discovered in different Burkholderia and Paraburkholderia species, be they members

398 or not of the Burkholderia cepacia complex (Bcc) (i.e. most pathogenic Burkholderia)

399 (Esmaeel et al., 2016). In our study, the ESI-MS analysis revealed that the major

400 metabolite in the purified extract (i.e., peak 1 with $\mathrm{RT}=1.2 \mathrm{~min}$ ) had an exact mass of

$401 \mathrm{MS}_{\mathrm{S}}=622.3069$ and $\mathrm{m} / \mathrm{z}=623.3069$ without iron and of $\mathrm{MFS}_{\mathrm{FS}}=675.2191$ with iron (Fig.

402 4B). This compound effectively chelated iron as stated with the CAS assay. This

403 mass value obtained with iron corresponds perfectly with the mass of the

404 malleobactin previously identified in the strains Burkholderia xenovorans LB400

405 (Vargas-Straube et al., 2016) and Burkholderia thailandensis (Franke et al., 2013). In both 
406 studies, the molecule detected was named malleobactin, but Vargas-Straube et al.

407 (2016) proposed the name malleobactin X and Franke et al. (2013) proposed

408 malleobactin $B$. The single difference between malleobactins $B$ and $X$ is the position

409 of an aldehyde group on the $\mathrm{N}$-terminal ornithine. Such position was predicted by

410 Vargas-Straube et al., (2016) to be on the second nitrogen of the N-ter ornithine,

411 while Franke et al., (2013) determined it by NMR to be on the first nitrogen of the N-

412 ter ornithine. As the two molecules present the same properties (i.e., mass,

413 fragmentation peaks, chelation ability) and the genome sequences are quite similar,

414 malleobactin B and X may correspond to the same molecule. For clarity in the text

415 we used the term malleobactin X. Finally, Vargas-Straube et al. (2016) also identified

416 another compound in the MS spectrum of the malleobactin produced by $B$.

417 xenovorans LB400 presenting a peak at $\mathrm{m} / \mathrm{z}=606.4$ that they hypothesized to be a

418 fragment of the ferrisiderophore produced by a McLafferty rearrangement

419 (elimination of $\mathrm{CH}_{2} \mathrm{CHCH}_{2} \mathrm{CH}_{2} \mathrm{NH}_{2}$ corresponding to [MFs-70] ${ }^{+}$), but without the

420 demonstration of the presence of iron in this compound. For our strain PMB3(1) of

421 Collimonas, we detected a similar compound $(\mathrm{m} / \mathrm{z}=606.2802)$ in the MS spectrum of

422 the ferrisiderophore (i.e., malleobactin $+\mathrm{Fe}$ ). However, the isotopic profile of this ion 25 
$423(\mathrm{~m} / \mathrm{z}=606.2802)$ did not present the characteristic iron signature, i.e. the clear

424 contributions of the two major iron isotopes ${ }^{54} \mathrm{Fe}$ and ${ }^{56} \mathrm{Fe}$ (Fig.S3). The absence of

425 iron signal indicates that the $\mathrm{m} / \mathrm{z}=606.2802$ molecule is consequently not the result

426 of a Mc Lafferty rearrangement of the ferrisiderophore.

428 The chemical analyses granted us the possibility to authenticate two other minor

429 peaks (2 and 3; Fig. 3) in the purified extract, far less intense than the peak 1

430 corresponding to malleobactin $(R T=1.2 \mathrm{~min})$. The absence of these two peaks in the

431 HPLC chromatogram of the extract of the $\triangle$ NRPS mutant (Fig. 3A) demonstrated that

432 the NRPS region we identified was responsible for the synthesis of (at least) the

433 three molecules (corresponding to the main peak 1 and the minor peaks 2 and 3).

434 Interestingly, the production of three different malleobactins by a single NRPS region

435 was also proposed for Burkholderia pseudomallei strain K96243, but the exact

436 structure and identity of these malleobactins were not reported (Alice et al., 2006). In

437 the current work, we show that these two other molecules presented a lower

438 chelating activity than the main molecule we identified as malleobactin X. This is not

439 incongruent with these molecules being malleobactins. In fact, among the wide range 
440 of malleobactins described (malleobactin A-H and X; (Franke et al., 2015; Vargas-

441 Straube et al., 2016), the affinity for iron strongly varies. Some of them (i.e.

442 malleobactin $B, F$ and $H$ ) present such a low chelating activity compared to

443 malleobactin $\mathrm{E}$ that their real role in cell has been questioned (Franke et al., 2015).

444 Thus diversity of malleobactin forms was proposed to result from the low specificity of

445 the adenylation domain A1 and A4 of the NRPS modules (Misiek et al., 2011; Franke et

446 al., 2015), but also from a spontaneous structural change (Franke et al., 2013).

447 Noticeably, malleobactin B (similar to malleobactin X) was shown to undergo such a

448 structural change to other malleobactin forms (A, C or D) without any enzyme activity

449 (Franke et al., 2013). Comparing the affinity for iron of these different forms revealed

450 that malleobactin A has a very low affinity. Based on the literature, our observations

451 suggest that the two minor peaks (2 and 3 ) represent alternate structures of the main

452 malleobactin detected in peak 1. However, due to their low concentration and low

453 affinity for iron, we were not able to determine their mass.

454

455 While the chemical characterization of the siderophore produced by strain

456 PMB3(1) allowed the identification of a malleobactin, the bioinformatic analyses done 
457 on the NRPS genomic sequence (antiSMASH) predicted the production of ornibactin

458 (Stephan et al., 1993). This discrepancy is mostly explained by the antiSMASH

459 database that does not contain malleobactin standard regions as few malleobactins

460 have been characterized at both chemical and genomic levels. The comparison of

461 the chemical structures of ornibactin and malleobactin reveals that they are strongly

462 similar (Alice et al., 2006; Deng et al., 2017). Indeed, the two siderophores differ only on

463 the N-terminal ornithine residue, where an aliphatic chain is present only on

464 ornibactin (Franke et al., 2013). Such similarities suggest that the two siderophores are

465 encoded by very similar NRPS systems and that many predicted ornibactin-

466 producing bacteria may be malleobactin producers. To solve this issue of

467 identification, Esmaeel et al. (2016) proposed to add new criteria to differentiate the

468 NRPS-encoding ornibactin and malleobactin based on particular genes present or

469 absent in the NRPS clusters (Esmaeel et al., 2016). They suggested that orbK-like and

470 orbL-like genes (encoding acetylases) are only present in ornibactin clusters,

471 whereas the presence of $m b a M$ gene (encoding a protein of unknown function) was

472 only present in malleobactin clusters (Esmaeel et al., 2016). However, the presence of

473 the mbaM gene was only highlighted for Burkholderiales (Butt and Thomas, 2017). In 
474 our study, we do not detect a mbaM homologue in our model strain nor in the other

475 Collimonas strains or in Burkholderia xenovorans strain LB400, indicating that this

476 protein is not necessary for malleobactin production and is not a good marker of the

477 malleobactin cluster. The absence of the orbK-like and orbL-like genes are therefore

478 the most relevant for the identification of the malleobactin cluster.

480 Siderophores represent one of the main class of molecules reported to play an

481 important role in mineral weathering which are frequently detected in soil (Holmström

482 et al., 2004). They are suspected to act directly on the mineral surfaces where they

483 form iron-siderophore complexes that are then transferred into the soil solution

484 (Kalinowski et al., 2000; Kraemer, 2004). They may also indirectly act in MW by

485 chelating the iron released in solution, a phenomenon that modifies the equilibrium of

486 the surrounding solution and thus enhances mineral dissolution. While the effect of

487 pure molecules was clearly evidenced, the experiments done with bacteria producing

488 such molecules were less clear. Indeed, concomitantly to the production of 489 siderophores, many bacteria acidify their environment, making it difficult to

490 disentangle the relative effects of the acidification and the chelating molecules in the 29 
491 mineral weathering process (Hersman et al., 1995; Holmén and Casey, 1996; Maurice,

492 2000). Furthermore, several studies have shown a synergistic effect of siderophores

493 and acidifying molecules on mineral weathering (Reichard et al., 2007; Dehner et al.,

494 2010; Lin et al., 2018). Controlling the $\mathrm{pH}$ of the solution is therefore essential at

495 chemical, mineralogical and microbiological levels. First, it conditions the activity of

496 the siderophores. Deferrioxamine B, protochelin or rhizoferrin clearly showed

497 contrasted chelating activity with $\mathrm{pH}$ (Akafia et al., 2014). Second, acidic conditions

498 allow a spontaneous dissolution of minerals (Brantley, 2008), which may release

499 significant quantities of iron in solution and consequently inhibit siderophore

500 production by bacteria. Indeed, the production of siderophore was shown to be

501 inhibited by iron concentrations ranging from 0.2 to $5 \mathrm{mg} / \mathrm{l}$ (Dave and Dubc, 2000; Deng

502 et al., 2017) or in presence of Fe-bearing glasses (Perez et al., 2019). In our study,

503 the production of malleobactin by strain PMB3(1) appeared inhibited from $0.8 \mathrm{mg} / \mathrm{l}$ of

504 iron in the solution, while the amount of iron released passively in solution from

505 hematite in our experimental conditions was below $0.1 \mathrm{mg} / \mathrm{l}$. To exclude a potential

506 effect of acidification, our mineral weathering experiments were performed in a

507 buffered medium (constant $\mathrm{pH}=6.2$ ), devoid of iron and containing a carbon source 
508 (i.e. mannitol), which does not permit an acidifying metabolism. Using this approach,

509 we were able to demonstrate for the first time that malleobactin production by

510 Collimonas pratensis strain PMB3(1) contribute to a significant increase of the

511 hematite dissolution as showed by comparing the WT and $\triangle$ NRPS mutant strains.

512 Together, our results suggest that depending on the availability of carbon substrates

513 (i.e., glucose vs mannitol) and the nutrients (i.e., quantity of iron in solution), strain

514 PMB3(1) may use high levels of organic acid such as gluconate and 2-keto-

515 gluconate or malleobactin to weather minerals and to scavenge iron from its

516 surrounding environment, two mechanisms which can act independently or in a

517 synergistic way (Picard et al., 2021).

518

519 CONCLUSION

520 The combination of molecular and chemical methods allowed us to identify the

521 siderophore produced by Collimonas pratensis strain PMB3(1) as malleobactin X.

522 This is the first chemical and molecular characterization of a siderophore produced

523 by a collimonads as previous studies were only based on in silico analyses which

524 predicted that the siderophore produced by Collimonas was ornibactin (Song et al., 
525 2015). Malleobactin is a hydroxamate type siderophore characterized by a large

526 diversity of structures that was previously reported in Burkholderiales and for several

527 strains of Burkholderia. The conservation of the NRPS region among Collimonas

528 species and the ability of the related strains to mobilize iron indicate that the

529 production of malleobactin is a common trait among collimonads. It would be

530 interesting to extend such analysis to a broader number of collimonads and

531 neighboring genera. This study is also the first one demonstrating that malleobactin

532 production is involved in mineral weathering. The use of a non-acidifying $\mathrm{C}$ source

533 and of buffered conditions clearly proved the impact of malleobactin on the

534 dissolution of hematite. Although malleobactin impacted hematite, a highly

535 weatherable iron oxide, its impact on primary minerals present in soils such as

536 phyllosilicate remains to be tested.

538 EXPERIMENTAL PROCEDURES

540 Bacterial strains and growth media 
541 Bacterial strains, plasmids and primers are listed in Table 1. The model Collimonas

542 pratensis strain PMB3(1) studied here was isolated from oak (Quercus petraea)-

543 Scleroderma citrinum ectomycorrhizae sampled in the organo-mineral soil horizon

544 from the long term experimental forest site of Breuil-Chenue (Morvan region, France;

545 for sampling details see (Calvaruso et al., 2007). This model strain was chosen for its

546 high mineral weathering effectiveness (Uroz et al., 2009b, 2007). All strains were grown

547 at $25^{\circ} \mathrm{C}$, except the strains of Escherichia coli, which were grown at $37^{\circ} \mathrm{C}$. The media

548 used were Luria-Bertani (LB) and AB medium supplemented with mannitol $(2 \mathrm{~g} / \mathrm{l}$ final

549 concentration) as carbon source (ABm; (Chilton et al., 1974). Gentamycin was added

550 to the media at $20 \mu \mathrm{g} / \mathrm{ml}$ (final concentration) when required.

551

552 Preparation of bacterial inocula

553 For each growth or functional assay presented below, the wild type (WT) strain

554 PMB3(1) and the mutant strain affected in its ability to mobilize iron ( $\triangle$ NRPS) were

555 recovered from glycerol stock $\left(-80^{\circ} \mathrm{C}\right)$ and grown on $\mathrm{ABm}$ solid medium, amended

556 with gentamycin for the $\triangle$ NRPS mutant. After a 2-day incubation at $25^{\circ} \mathrm{C}$, one colony

557 of each strain was inoculated in liquid $\mathrm{ABm}$ medium $(10 \mathrm{ml})$ and incubated for 2 days 33 
558 at $25{ }^{\circ} \mathrm{C}$ under $200 \mathrm{rpm}$ agitation. The cultures were then centrifuged at $8,000 \mathrm{~g}$ for

$55915 \mathrm{~min}$ at $10^{\circ} \mathrm{C}$ and the pellet was washed twice with $5 \mathrm{ml}$ of sterile Milli-Q water to

560 eliminate all culture medium and antibiotic traces. The pellet was recovered in $5 \mathrm{ml}$ of

561 sterile Milli-Q water. To control the quantity of cells inoculated, the optical density

562 (OD) was measured at $590 \mathrm{~nm}$ and cell suspensions were adjusted at $0.90 \cdot 0.03$.

563

\section{Siderophore activity}

565 To determine the siderophore activity, the Chrome Azurol S (CAS) assay was

566 performed according to (Schwyn and Neilands, 1987) using the solid and liquid

567 versions. Both assays are based on the competition for iron between the CAS-Fe(III)

568 complex and siderophores. In absence of chelating agent, iron is associated to the

569 Chrome Azurol $S$ and forms a blue complex. When a siderophore with a higher

570 affinity for iron is present, iron is released from the CAS-Fe(III) complex and the

571 colour of the CAS solution turns to yellow.

572 For the solid CAS assay, a volume of $5 \mu \mathrm{l}$ of the bacterial inoculum of the WT or

573 the mutant strains were inoculated on CAS agar plates and incubated at $25{ }^{\circ} \mathrm{C}$ for 3 
574 days. The presence of yellow halo was scored and its diameter as well as that of the

575 colony diameter was measured, to determine siderophore production and the growth.

576 For the liquid CAS assay, a volume of $100 \mu \mathrm{l}$ of the bacterial inoculum of the WT

577 or the mutant strains were inoculated in liquid $\mathrm{ABm}$ medium devoid of iron (10 ml)

578 and amended with gentamycin for the mutant strain. After 2-days incubation at $25^{\circ} \mathrm{C}$,

579 the cultures were centrifugated at $8,000 \mathrm{~g}$ for $15 \mathrm{~min}$ at $10{ }^{\circ} \mathrm{C}$ to recover the culture

580 supernatant. The supernatant was then filtered at $0.22 \mu \mathrm{m}$ (GHP Acrodisc $25 \mathrm{~mm}$

581 syringe filter; PALL) and stored at $-20{ }^{\circ} \mathrm{C}$ for further analyses. To evaluate the

582 presence of siderophore activity, $100 \mu \mathrm{l}$ of the filtered supernatant was added to

$583100 \mu \mathrm{l}$ of liquid CAS medium devoid of carbon source in a 96 -well microplate. The

584 supernatant of non-inoculated medium was used as a negative control. After 1 hour

585 of incubation at room temperature in the dark, the absorbance was measured at OD

$586=655 \mathrm{~nm}$ with a microplate reader (Bio-Rad, model iMark). The decrease of

587 absorbance corresponds to a change from blue color of the CAS complex to a yellow

588 color when a siderophore is present. 
591 Total DNA was extracted from the WT strain using the protocol of (Pospiech and

592 Neumann, 1995). To construct a targeted mutant of the gene $m b a A$, a portion of this

593 gene was amplified using the primers For-NRPS and Rev-NRPS (Table 1). These

594 primers amplified a region of ca. $2 \mathrm{~kb}$ of the $m b a A$ gene characterized by the

595 presence of a natural Smal site in its center. The purified PCR product was ligated

596 into the pGEM-T Easy plasmid (Promega) resulting in the plasmid pGEM-NRPS

597 (Table 1). The plasmid pGEM-NRPS was then digested with Smal to introduce the

598 Smal-digested gentamycin $(\mathrm{Gm})$ resistance cassette from plasmid pUC1318,

599 resulting in the plasmid pGEM-NRPS::Gm. A fragment containing the mbaA::Gm

600 cassette was then obtained by EcoRI restriction and cloned in the plasmid pK19mob,

601 resulting in the pK19mob-NRPS::Gm plasmid. This construction was transferred to $E$.

602 coli S17.1 $\lambda$ pir and then to strain PMB3(1) by bi-parental conjugation. $\triangle$ NRPS mutants

603 were recovered after 5 days of incubation at $25^{\circ} \mathrm{C}$ on gentamycin-containing $A B m$

604 plates and organized in 96-microtiter plates. The transconjugants obtained were

605 verified by PCR with For-NRPS and Rev-NRPS primers, to differentiate the single

606 and double crossing-over events. Several single and double crossing-over mutants 
607 were conserved. The experiments described in our study were done using a double

608 crossing-over mutant named $\triangle$ NRPS.

609

610 Minimal concentration of iron inhibiting siderophore production

611 To evaluate the concentration of iron inhibiting the siderophore production, the WT

612 strain and its $\triangle$ NRPS mutant were incubated with different concentrations of iron.

613 Briefly, in a 96-wells microplate, $10 \mu \mathrm{l}$ of calibrated suspension (described above) of

614 WT and $\triangle$ NRPS were inoculated in $180 \mu \mathrm{l}$ of ABm medium devoid of iron and $10 \mu \mathrm{l}$ of

615 a $\mathrm{FeCl}_{3}$ solution. Different $\mathrm{FeCl}_{3}$ solutions were prepared in Milli-Q water to obtain

616 final concentrations of iron ranging from 0 to $5 \mathrm{mg} / \mathrm{l}(0 ; 0.1 ; 0.2 ; 0.3 ; 0.5 ; 0.6 ; 0.7 ; 0.8$;

$6170.9 ; 1.0 ; 1.5 ; 2.0 ; 2.5 ; 5.0 \mathrm{mg} / \mathrm{l})$. A non-inoculated condition was performed as control.

618 After 3 days of incubation, the 96 -wells microplate was centrifuged (1,100 g; $15 \mathrm{~min})$

619 and $100 \mu \mathrm{l}$ of supernatant were transferred to a new 96-wells microplate and mixed

620 with $100 \mu$ l of CAS liquid solution (as described above). This solution was incubated

$6211 \mathrm{~h}$ in the dark before measuring the absorbance at $655 \mathrm{~nm}$. 
623 Impact of iron concentration and iron competition on the growth of strain PMB3(1)

\section{4 and its $\triangle$ NRPS mutant}

625 To determine how iron concentration affects the growth of the WT strain and its

$626 \triangle$ NRPS mutant, cultures were done in $\mathrm{ABm}$ medium devoid of iron and

627 supplemented or not with $\mathrm{FeCl}_{3}$ (final concentration of iron: $0 ; 0.1 ; 0.2 ; 0.3 ; 0.5 ; 1.0$

$628 \mathrm{mg} / \mathrm{l}$ ). A volume of $10 \mu \mathrm{l}$ each of bacterial inoculum (i.e., WT and mutant) was added

629 to $190 \mu \mathrm{l}$ each of $\mathrm{ABm}$ medium described above. In addition, a competition assay

630 was done to determine how iron availability may affect the growth of strain PMB3(1)

631 and its $\triangle$ NRPS mutant. To do it, a strong chelator agent (i.e., $10 \mathrm{mM}$ of EDTA) was

632 added or not to the culture medium supplemented with $1 \mathrm{mg} / \mathrm{l}$ of iron. All samples

633 were tested in triplicate. The 96 -well microplate was incubated at $25^{\circ} \mathrm{C}$ on orbital

634 shaking during 3 days in a microplate reader (Tecan infinite M200 pro). The

635 absorbance was measured at OD $=600 \mathrm{~nm}$ every 3 hours. To evaluate the growth of

636 the strains, the slope of the exponential phase (i.e. growth rate) was calculated for

637 each condition and replicate. 
641 To enable the chemical characterization of the siderophore(s) produced by strain

642 PMB3(1), cultures of $50 \mathrm{ml}$ were done in iron-depleted ABm culture medium. The WT

643 strain and its $\triangle$ NRPS mutant were incubated 3 days at $25{ }^{\circ} \mathrm{C}$ under shaking (200

$644 \mathrm{rpm})$. A non-inoculated condition was used as negative control. After this incubation

645 period, the cultures were centrifugated at $8,000 \mathrm{~g}$ for $15 \mathrm{~min}$ and the supernatant was

646 filtered at $0.22 \mu \mathrm{m}$ and stored at $-20{ }^{\circ} \mathrm{C}$ for further analyses. The siderophore was

647 concentrated and purified using a Sep pack Vac 6cc tC18 Cartridges (Waters). The

648 column was first washed with $10 \mathrm{ml}$ of $100 \%$ methanol, then it was equilibrated with

$64910 \mathrm{ml}$ of $0.01 \%$ trifluoroacetic acid (TFA) to acidify the column. Next, $25 \mathrm{ml}$ of

650 supernatant was applied on the column. The siderophore was eluted with $5 \mathrm{ml}$ of 10

$651 \%$ methanol solution (i.e. methanol / MQ water). The eluent was collected into $2 \mathrm{ml}$

652 eppendorf tubes and evaporated in a speedvacum system for $5 \mathrm{~h}$. The dried samples

653 were dissolved in $100 \mu \mathrm{l}$ of $\mathrm{MQ}$ water. The siderophore activity was tested using the

654 liquid CAS assay on the culture filtrate $(0.22 \mu \mathrm{m})$ as well as on the Sep pack purified

655 solution. 
658 A siderophore-containing extract was analyzed in the presence and in the absence

659 of an iron(III) acetate solution. Iron(III) solution was prepared with a 4-fold excess of 660 acetate relative to iron $(25 \mathrm{mM}) /$ acetate $(100 \mathrm{mM})$. Iron(III) chloride $\left(\mathrm{FeCl}_{3}\right)$ was

661 initially dissolved in glacial acetic acid before dilution with water to desired volume

$662(\mathrm{pH}=1.90)$ and finally double filtrated.

663 The formation of ferrisiderophore was carried out under non-inert atmosphere as

664 follows. Extract was first diluted 10 times $(\mathrm{pH}=4.01)$ and $\mathrm{pH}$ was adjusted at $\mathrm{pH} 2.79$

665 with $0.1 \mathrm{M} \mathrm{HCl}$. Then, iron(III) acetate solution $(25 \mathrm{mM}, 100 \mu \mathrm{l})$ was added to $400 \mu \mathrm{l}$ 666 of diluted extract to get the final reaction mixture $(500 \mu \mathrm{l})$, at $\mathrm{pH} 2.46$.

667 The control test was simply carried out by mixing $400 \mu$ of 10 -times diluted extract

668 with $100 \mu \mathrm{l}$ of pure water, $\mathrm{pH}$ being adjusted under $\mathrm{pH} 3$ with $0.1 \mathrm{M} \mathrm{HCl}$.

670 High Performance Liquid Chromatography (HPLC) analyses and fractionation

671 The analysis was done on Sep pack treated supernatants coming from inoculated

672 (WT and $\triangle$ NRPS strains) or non-inoculated (control medium) media. Fifty microliters

673 of each supernatant were injected onto a Gemini C18 column ( $150 \times 3.0 \mathrm{~mm}$ internal 
674 diameter, $5 \mu \mathrm{m}$ particle size, Phenomenex) equilibrated with Milli-Q water with $0.01 \%$

675 formic acid at $25^{\circ} \mathrm{C}$ and connected to a Shimadzu Prominence HPLC system. A

676 gradient elution of Milli-Q water with $0.01 \%$ formic acid (buffer $A$ ) and acetonitrile with

$6770.01 \%$ formic acid (buffer B) for $40 \mathrm{~min}$ was used at a flow-rate of $1 \mathrm{ml} / \mathrm{min}$ and

678 detection was monitored with diode array UV-vis detector at $210 \mathrm{~nm}$. The potential

679 peaks of interest were identified by comparing HPLC spectra and retention times

680 from the WT and $\triangle$ NRPS strains and the control medium samples.

681 To determine the siderophore activity of the peaks of interest, a fraction collect

682 was performed in 96-wells microplate using fraction collector system (FRC-10A

683 fraction collector system, Shimadzu). For each sample, fractions of $200 \mu$ were

684 recovered each $12 \mathrm{~s}$ using the same HPLC conditions described above. Fractions

685 were then evaporated in a speedvacum system. Samples were suspended in $25 \mu \mathrm{l}$ of

686 CAS liquid assay diluted with $25 \mu \mathrm{l}$ of Milli-Q water and transferred in a new

687 microplate for absorbance measure at $655 \mathrm{~nm}$.

688 To perform further mass spectrum analyses, the major peaks identified were

689 collected and the fractions combined to concentrate the metabolite of interest. 
692 (LC-ESI-HRMS)

693 Qualitative and semi-quantitative analysis of siderophores (S) and

694 ferrisiderophores (FS) was realized using a LC-ESI-HRMS method previously

695 described by (Paris et al., 2021). The UHPLC-MS system (ThermoFisher Scientific,

696 San Jose, CA, USA) consists in a quaternary UltiMate $3000^{\mathrm{TM}}$ solvent delivery pump

697 connected to a photodiode array detector (PDA) and a LTQ-Orbitrap TM hybrid mass

698 spectrometer. Ten microliters of pre-purified HPLC extract were separated on a C18

699 Alltima reverse phase column $(150 \times 2.1 \mathrm{~mm}, 5 \mu \mathrm{m}$ - Grace/Alltech, Darmstadt,

700 Germany) equipped with a C18 Altima pre-column $(7.5 \times 2.1 \mathrm{~mm}, 5 \mu \mathrm{m})$ at $25^{\circ} \mathrm{C}$.

701 The flow rate was set at $0.2 \mathrm{ml} / \mathrm{min}$ and mobile phases consisted in water modified

702 with nonafluoropentanoic acid (NFPA) $(5 \mathrm{mM})$ for $A$ and pure methanol for B.

703 Compounds of interest were separated thanks to a program of $36 \mathrm{~min}$, using a linear

704 gradient from $5 \%$ to $98 \%$ of $\mathrm{B}$ for $25 \mathrm{~min}$.

705 Mass analysis was carried out in ESI positive ion mode $\left(\mathrm{ESI}^{+}\right)$and mass

706 spectrometry conditions were as follows: spray voltage was set at $4.5 \mathrm{kV}$; source

707 gases were set (in arbitrary units/min) for sheath gas, auxiliary gas and sweep gas at 
70840,5 and 5 , respectively; capillary temperature was set at $300^{\circ} \mathrm{C}$; capillary voltage at

$70946 \mathrm{~V}$; tube lens, split lens and front lens voltages at $112 \mathrm{~V},-70 \mathrm{~V}$ and $-6.25 \mathrm{~V}$,

710 respectively.

711 Full scan MS spectra were performed at high resolution $(\mathrm{R}=60000$ at $\mathrm{m} / \mathrm{z} 400)$ on

712 the Orbitrap ${ }^{\mathrm{TM}}$ analyzer from 120 to $2000 \mathrm{~m} / \mathrm{z}$ to obtain exact masses of siderophores

713 and ferrisiderophores on the HPLC purified peaks as well as on the total extract. MS 2

714 full scan spectra were additionally realized for structural elucidation thanks to LTQTM

715 analyzer (Linear Trap Quadrupole). Raw data were processed using the

716 XCALIBUR ${ }^{T M}$ software program (version 2.1, http://www.thermoscientific. com).

\section{Hematite weathering assay}

719 Mineral description

720 Hematite used as mineral in our study comes from a batch isolated in Brazil.

721 Hematite is an iron-oxide mineral of the oxides and hydroxides group. Iron oxides

722 (i.e., hematite, goethite) occur, at least in small amounts, in nearly all soils (Dixon et

723 al., 1989). Hematite is a widespread mineral occurring in a great variety of soils as a

724 result of the weathering of Fe-containing minerals (Dixon et al., 1989). Igneous rocks 43 
725 (i.e., granite, trachyte, and rhyolite) can contain primary accessory grains of hematite.

726 This mineral was chosen for its high weatherability, its high content of iron and the

727 presence of hematite as secondary minerals in the experimental site where was

728 isolated the strain PMB3(1) (Calvaruso et al., 2009). In this study, pure hematite

729 crystals were used $\left(\mathrm{Fe}_{2} \mathrm{O}_{3}\right.$ pure at $\left.99.3 \%\right)$ and containing in small amounts: $0.40 \%$

$730 \mathrm{TiO}_{2}, 0.18 \% \mathrm{SiO}_{2}, 0.16 \% \mathrm{Al}_{2} \mathrm{O}_{3}, 0.10 \% \mathrm{MgO}, 0.037 \% \mathrm{MnO}$.

731

732 Mineral weathering assay

733 The assay was performed in glass tubes containing $2 \mathrm{~g} / \mathrm{l}$ of sterile hematite and

$73410 \mathrm{ml}$ of $\mathrm{ABm}$ medium devoid of iron. Before introduction of hematite, the glass tubes

735 were rinsed with hydrochloric acid $(\mathrm{HCl}, 3.6 \%)$ and MilliQ water. The tubes

736 containing the culture medium and hematite were then sterilised by autoclaving at

$737121^{\circ} \mathrm{C}$. For the weathering assay, $100 \mu \mathrm{l}$ of each bacterial inoculum (described

738 above; WT and $\triangle$ NRPS mutant strains) were used to inoculate the sterile glass

739 tubes. Non-inoculated media with and without hematite were used as controls. After

740 seven days of incubation at $25{ }^{\circ} \mathrm{C}(200 \mathrm{rpm}), 200 \mu \mathrm{l}$ of supernatant were sampled

741 and the OD at $595 \mathrm{~nm}$ was measured to determine bacterial growth. Then, $1 \mathrm{ml}$ of 
742 supernatant was centrifuged at $11,000 \mathrm{~g}$ for $15 \mathrm{~min}$ to remove bacterial cells.

743 Quantification of the iron released from hematite in the culture supernatant as well as

744 the $\mathrm{pH}$ were determined by adding $180 \mu \mathrm{l}$ of supernatant of each culture with $20 \mu \mathrm{l}$ of

745 ferrospectral or bromocresol green (1 $\mathrm{g} / \mathrm{l})$, respectively (Uroz et al., 2007). The

746 absorbance of these suspensions was measured at $595 \mathrm{~nm}$. The same culture

747 supernatant was also used to measure siderophore production with the liquid CAS

748 assay. The average values for three independent replicates for growth, iron

749 quantification, $\mathrm{pH}$ and siderophore production were used to determine the weathering

750 potential of the $\triangle$ NRPS mutant and the wild-type strains.

\section{Bioinformatic analyses}

753 Genome properties gene localization and homology

754 Genome analysis was performed using NCBI and MaGe (Vallenet, 2006). Homology

755 search analysis and alignment were performed with BLAST (BlastN and BlastP) (Altschul et 756 al., 1990).

757 In silico search and identification of NRPS genes.

758 An in silico survey was done on the genome sequence of strain PMB3(1) to

759 identify the conserved domains of NRPS involved in siderophore production using 
760 antiSMASH (Blin et al., 2019). A combination of 3 tools based on the Stachelhaus

761 code of the adenylation domain was used to predict the amino acids recruited and the

762 potential structure of the siderophore produced (NRPS predictor (Rausch, 2005);

763 PKS/NRPS Analysis (Ansari et al., 2004); NP.searcher (Li et al., 2009)).

764

765

Phylogenetic analysis on Collimonas genus

766 As the MbaA protein is involved in the synthesis of the most variable part (i.e. $\mathrm{N}$ -

767 terminal part) of the siderophore, a phylogenetic analysis was performed on it (Franke

768 et al., 2015). A total of 52 NRPS protein sequences were used to build a phylogenetic

769 PhyML tree with Seaview (version 4.5.4; (Gouy et al., 2010)). These sequences were

770 chosen for their similarity to Orbl or MbaA proteins that are NRPS responsible of

771 ornibactin or malleobactin biosynthesis, respectively. Our analysis includes

772 sequences from Collimonas genera available online and other Collimonas related

773 genera (i.e. Paraburkholderia, Burkholderia, Caballeronia, Herbaspirillum) for which

774 the siderophores produced were predicted or experimentally demonstrated (Table

775 S1). As Collimonas is strongly related to Herbaspirillum, the NRPS protein involved in 
776 serobactin production by Herbaspirillum seropedicae was included (Rosconi et al.,

777 2013).

778

779 Statistical analyses.

780 Statistical analyses were performed in R software. Data shown were means of at

781 least triplicates. Differences between sample's means were analysed by ANOVA and

782 TukeyHSD tests.

783

784 Nucleotide sequence accession number.

785 The whole-genome is available on NCBI under the accession no. WXXL01000000.

786

787 ACKNOWLEDGMENTS

788 This work was supported by grants from the EC2CO program of the CNRS to S.U.

789 and P.O. L.P. was also supported by a fellowship from the French Ministère de

790 l'Enseignement Supérieur, de la Recherche et de l'Innovation. The UMR1136 and

791 UR1138 are supported by the ANR through the Laboratory of Excellence Arbre

792 (ANR-11-LABX-0002-01). 


\section{REFERENCES}

795 Agnoli, K., Lowe, C.A., Farmer, K.L., Husnain, S.I., and Thomas, M.S. (2006) The ornibactin biosynthesis and transport genes of Burkholderia cenocepacia are regulated by an extracytoplasmic function factor which is a part of the Fur regulon. J Bacterio/ 188: 3631-3644.

Akafia, M.M., Harrington, J.M., Bargar, J.R., and Duckworth, O.W. (2014) Metal oxyhydroxide dissolution as promoted by structurally diverse siderophores and oxalate. Geochim Cosmochim Acta 141: 258-269.

Alice, A.F., Lopez, C.S., Lowe, C.A., Ledesma, M.A., and Crosa, J.H. (2006) Genetic and transcriptional analysis of the siderophore malleobactin biosynthesis and transport genes in the human pathogen Burkholderia pseudomallei K96243. J Bacteriol 188: 1551-1566.

806 Altschul, S.F., Gish, W., Miller, W., Myers, E.W., and Lipman, D.J. (1990) Basic Local Alignment Search Tool. J Mol Bio/215: 403-410.

808 Andrews, S.C. (1998) Advances in microbial physiology, San Diego, Calif.; London: Academic. 
Ansari, Mohd.Z., Yadav, G., Gokhale, R.S., and Mohanty, D. (2004) NRPS-PKS: a knowledge-based resource for analysis of NRPS/PKS megasynthases. Nucleic Acids Res 32: W405-W413.

813 Ballhausen, M.-B., Vandamme, P., and de Boer, W. (2016) Trait differentiation within the fungus-feeding (mycophagous) bacterial genus Collimonas. PLOS ONE 11: e0157552.

816 Blin, K., Shaw, S., Steinke, K., Villebro, R., Ziemert, N., Lee, S.Y., et al. (2019) antiSMASH 5.0: updates to the secondary metabolite genome mining pipeline. Nucleic Acids Res 47: W81-W87.

819 Brantley, S.L. (2008) Kinetics of Mineral Dissolution. In Kinetics of Water-Rock Interaction. Brantley, S.L., Kubicki, J.D., and White, A.F. (eds). New York, NY: Springer, pp. 151-210.

822 Braun, V., Mahren, S., and Ogierman, M. (2003) Regulation of the Fecl-type ECF sigma factor by transmembrane signalling. Curr Opin Microbio/6: 173-180.

824 Butaitè, E., Baumgartner, M., Wyder, S., and Kümmerli, R. (2017) Siderophore cheating and cheating resistance shape competition for iron in soil and freshwater Pseudomonas communities. Nat Commun 8: 414. 
827 Butt, A.T. and Thomas, M.S. (2017) Iron acquisition mechanisms and their role in the $828 \quad$ virulence of Burkholderia species. Front Cell Infect Microbiol 7:

829 Calvaruso, C., Mareschal, L., Turpault, M.-P., and Leclerc, E. (2009) Rapid clay weathering in the rhizosphere of norway spruce and oak in an acid forest ecosystem. Soil Sci Soc Am J 73: 331-338.

832 Calvaruso, C., Turpault, M.-P., Leclerc, E., and Frey-Klett, P. (2007) Impact of ectomycorrhizosphere on the functional diversity of soil bacterial and fungal communities from a forest stand in relation to nutrient mobilization processes. Microb Eco/54: 567-577.

836 Chilton, M.-D., Currier, T.C., Farrand, S.K., Bendich, A.J., Gordon, M.P., and Nester, E.W. (1974) Agrobacterium tumefaciens DNA and PS8 bacteriophage DNA not detected in crown gall tumors. Proc Natl Acad Sci 71: 3672-3676.

839 Darling, P., Chan, M., Cox, A.D., and Sokol, P.A. (1998) Siderophore production by cystic fibrosis isolates of Burkholderia cepacia. Infect Immun 66: 874-877.

841 Dave, B.P. and Dubc, H.C. (2000) Regulation of siderophore production by iron $\mathrm{Fe}(\mathrm{III})$ in certain fungi and fluorescent pseudomonads. Indian $J$ Exp Bio/ 38: 297-299. 
844 De Boer, W. (2004) Collimonas fungivorans gen. nov., sp. nov., a chitinolytic soil bacterium with the ability to grow on living fungal hyphae. Int J Syst Evol Microbio/ 54: 857-864.

847 Dehner, C.A., Awaya, J.D., Maurice, P.A., and DuBois, J.L. (2010) Roles of 848 siderophores, oxalate, and ascorbate in mobilization of iron from hematite by 2041-2048.

851 Deng, P., Foxfire, A., Xu, J., Baird, S.M., Jia, J., Delgado, K.H., et al. (2017) The siderophore product ornibactin Is required for the bactericidal activity of Burkholderia contaminans MS14. App/ Environ Microbio/83:.

854 Dixon, J.B., Weed, S.B., and Dinauer, R.C. eds. (1989) Minerals in soil environments, 2nd ed. Madison, Wis., USA: Soil Science Society of America.

856 Doan, H.K., Maharaj, N.N., Kelly, K.N., Miyao, E.M., Davis, R.M., and Leveau, J.H.J. (2020) Antimycotal activity of Collimonas isolates and synergy-based biological control of $<\mathrm{i}>$ Fusarium<i/> wilt of tomato. Phytobiomes J 4: 64-74.

859 Dong, H. (2010) Mineral-microbe interactions: a review. Front Earth Sci China 4: 
861 Esmaeel, Q., Miotto, L., Rondeau, M., Leclère, V., Clément, C., Jacquard, C., et al. (2018) Paraburkholderia phytofirmans PsJN-plants interaction: from perception to the induced mechanisms. Front Microbio/9: 2093.

864 Esmaeel, Q., Pupin, M., Kieu, N.P., Chataigné, G., Béchet, M., Deravel, J., et al. (2016) Burkholderia genome mining for nonribosomal peptide synthetases reveals a great potential for novel siderophores and lipopeptides synthesis. MicrobiologyOpen 5: 512-526.

868 Franke, J., Ishida, K., and Hertweck, C. (2015) Plasticity of the malleobactin pathway and its impact on siderophore action in human pathogenic bacteria. Chem Eur J 21: 8010-8014.

871 Franke, J., Ishida, K., Ishida-Ito, M., and Hertweck, C. (2013) Nitro versus hydroxamate in siderophores of pathogenic bacteria: effect of missing hydroxylamine protection in malleobactin biosynthesis. Angew Chem Int Ed 52: $8271-8275$.

875 Gouy, M., Guindon, S., and Gascuel, O. (2010) SeaView version 4: A multiplatform graphical user interface for sequence alignment and phylogenetic tree building. Mol Biol Evol 27: 221-224. 
878 Hernandez, M.E., Kappler, A., and Newman, D.K. (2004) Phenazines and other

879 redox-active antibiotics promote microbial mineral reduction. Appl Environnemental Microbio/ 70: 8.

881 Hersman, L., Lloyd, T., and Sposito, G. (1995) Siderophore-promoted dissolution of hematite. Geochim Cosmochim Acta 59: 3327-3330.

883 Holden, M.T.G., Seth-Smith, H.M.B., Crossman, L.C., Sebaihia, M., Bentley, S.D., 884 Cerdeño-Tárraga, A.M., et al. (2009) The genome of Burkholderia 885 cenocepacia J2315, an epidemic pathogen of cystic fibrosis patients. $J$ Bacterio/ 191: 261-277.

887 Holmén, B.A. and Casey, W.H. (1996) Hydroxamate ligands, surface chemistry, and 888 the mechanism of ligand-promoted dissolution of goethite [a-FeOOH(s)]. Geochim Cosmochim Acta 60: 4403-4416.

890 Holmström, S.J.M., Lundström, U.S., Finlay, R.D., and van Hees, P.A.W. (2004) Siderophores in forest soil solution. Biogeochemistry 71: 247-258.

892 Hoppener-Ogawa, S., de Boer, W., Leveau, J.H.J., van Veen, J.A., de Brandt, E., 

Microbio/58: 414-419.

896 Kalinowski, B.E., Liermann, L.J., Brantley, S.L., Barnes, A., and Pantano, C.G. (2000) X-ray photoelectron evidence for bacteria-enhanced dissolution of hornblende. Geochim Cosmochim Acta 64: 1331-1343. unpredicted in vitro but efficient in vivo biocontrol agent for the suppression of tomato foot and root rot. Environ Microbio/ 9: 1597-1603.

902 Koele, N., Turpault, M.-P., Hildebrand, E.E., Uroz, S., and Frey-Klett, P. (2009) Interactions between mycorrhizal fungi and mycorrhizosphere bacteria during mineral weathering: Budget analysis and bacterial quantification. Soil Biol Biochem 41: 1935-1942.

906 Kraemer, S.M. (2004) Iron oxide dissolution and solubility in the presence of siderophores. Aquat Sci - Res Boundaries 66: 3-18.

908 Lepleux, C., Turpault, M.P., Oger, P., Frey-Klett, P., and Uroz, S. (2012) Correlation of the abundance of betaproteobacteria on mineral surfaces with mineral weathering in forest soils. App/ Environ Microbio/78: 7114-7119. 
911 Leveau, J.H.J., Uroz, S., and de Boer, W. (2010) The bacterial genus Collimonas : mycophagy, weathering and other adaptive solutions to life in oligotrophic soil environments. Environ Microbio/ 12: 281-292.

914 Li, M.H., Ung, P.M., Zajkowski, J., Garneau-Tsodikova, S., and Sherman, D.H. 915 (2009) Automated genome mining for natural products. BMC Bioinformatics 10: 185 .

917 Lin, Q., Wang, Y., Yang, X., Ruan, D., Wang, S., Wei, X., and Qiu, R. (2018) Effect of 918 low-molecular-weight organic acids on hematite dissolution promoted by 919 desferrioxamine B. Environ Sci Pollut Res 25: 163-173.

920 Lindsay, W.L. (1995) Chemical reactions in soils that affect iron availability to plants. A quantative approach. In Iron Nutrition in Soils and Plants. Abadía, J. (ed). Dordrecht: Springer Netherlands, pp. 7-14.

923 Manura, J.J. and Manura, D.J. (2015) Isotope Distribution Calculator and Mass Spec $924 \quad$ Plotter, Scientific Instrument Services (SIS).

925 Mathew, A., Eberl, L., and Carlier, A.L. (2014) A novel siderophore-independent 926 strategy of iron uptake in the genus Burkholderia: Siderophore-independent iron uptake in Burkholderia. Mol Microbio/91: 805-820. 
928 Matzanke, B.F., Anemüller, S., Schünemann, V., Trautwein, A.X., and Hantke, K.

929 (2004) FhuF, part of a siderophore-reductase system †. Biochemistry 43:

$930 \quad 1386-1392$.

931 Maurice, P.A. (2000) Dissolution of Al-substituted goethites by an aerobic Pseudomonas mendocina var. bacteria. Geochim Cosmochim Acta 64: 13631374.

934 McMorran, B.J., Sullivan, K., and Lamont, I.L. (2001) Involvement of a transformylase enzyme in siderophore synthesis in Pseudomonas aeruginosa. Microbiology $1517-1524$.

937 Meyer, J.-M., Van Van, T., Stintzi, A., Berge, O., and Winkelmann, G. (1995) 938 Ornibactin production and transport properties in strains of Burkholderia 939 vietnamiensis and Burkholderia cepacia (formerly Pseudomonas cepacia).

$940 \quad$ Biometals 8: 309-317.

941 Misiek, M., Braesel, J., and Hoffmeister, D. (2011) Characterisation of the ArmA adenylation domain implies a more diverse secondary metabolism in the genus Armillaria. Fungal Biol 115: 775-781. 
944 Nienaber, A., Hennecke, H., and Fischer, H.-M. (2002) Discovery of a haem uptake system in the soil bacterium Bradyrhizobium japonicum: Haem uptake system in B. japonicum. Mol Microbio/41: 787-800.

947 Paris, C., Selmeczi, K., Ebel, B., Stefan, L., Csire, G., Cakir-Kiefer, C., et al. (2021) chelating peptides in protein hydrolysates. Anal Bioanal Chem.

950 Parrello, D., Zegeye, A., Mustin, C., and Billard, P. (2016) Siderophore-mediated iron Microbio/7:423.

953 Perez, A., Rossano, S., Trcera, N., Verney-Carron, A., Rommevaux, C., Fourdrin, C., ... \& Guyot, F. (2019). Direct and indirect impact of the bacterial strain Pseudomonas aeruginosa on the dissolution of synthetic Fe (III)-and Fe (II)bearing basaltic glasses. Chemical Geol, 523: 9-18.

957 Picard, L., Oger, P., Turpault, M.-P., and Uroz, S. (2020) Draft genome sequence of Collimonas pratensis strain PMB3(1), an effective mineral-weathering and chitin-hydrolyzing bacterial strain. Microbiol Resour Announc 9: e00601-20, /mra/9/37/MRA.00601-20.atom. 
961 Picard, L., Turpault, M.-P., Oger, P.M., and Uroz, S. (2021) Identification of a novel type of glucose dehydrogenase involved in the mineral weathering ability of Collimonas pratensis strain PMB3(1). FEMS Microbiol Ecolfiaa232.

964 Pospiech, A. and Neumann, B. (1995) A versatile quick-prep of genomic DNA from 965 gram-positive bacteria. Trends Genet TIG 11: 217-218.

966 Rausch, C. (2005) Specificity prediction of adenylation domains in nonribosomal peptide synthetases (NRPS) using transductive support vector machines (TSVMs). Nucleic Acids Res 33: 5799-5808.

969 Reichard, P.U., Kretzschmar, R., and Kraemer, S.M. (2007) Dissolution mechanisms of goethite in the presence of siderophores and organic acids. Geochim Cosmochim Acta 71: 5635-5650.

972 Rosconi, F., Davyt, D., Martínez, V., Martínez, M., Abin-Carriquiry, J.A., Zane, H., et

973 al. (2013) Identification and structural characterization of serobactins, a suite

974 of lipopeptide siderophores produced by the grass endophyte Herbaspirillum

15: 916-927. 
977 Rout, G.R. and Sahoo, S. (2015) Role of iron in plant growth and metabolism. Rev Agric Sci 3: 1-24.

979 Schwager, S., Agnoli, K., Köthe, M., Feldmann, F., Givskov, M., Carlier, A., and Eberl, L. (2013) Identification of Burkholderia cenocepacia strain H111 virulence factors using nonmammalian infection hosts. Infect Immun 81: 143153.

983 Schwyn, B. and Neilands, J.B. (1987) Universal chemical assay for the detection and 984 determination of siderophores. Anal Biochem 160: 47-56.

985 Senechkin, I.V., van Overbeek, L.S., Er, H.L., de Vos, O., and van Bruggen, A.H.C. (2013) Interaction of Collimonas strain IS343 with Rhizoctonia solani at low carbon availability in vitro and in soil. Eur J Plant Patho/136: 789-802.

988 Shirvani, M. and Nourbakhsh, F. (2010) Desferrioxamine-B adsorption to and iron dissolution from palygorskite and sepiolite. App/ Clay Sci 48: 393-397.

990 Song, C., Schmidt, R., de Jager, V., Krzyzanowska, D., Jongedijk, E., Cankar, K., et al. (2015) Exploring the genomic traits of fungus-feeding bacterial genus Collimonas. BMC Genomics 16: 1103-1120. 
993 Stachelhaus, T., Mootz, H.D., and Marahiel, M.A. (1999) The specificity-conferring 994 code of adenylation domains in nonribosomal peptide synthetases. Chem Biol 995 6: 493-505.

996 Stephan, H., Freund, S., Beck, W., Jung, G., Meyer, J.-M., and Winkelmann, G. (1993) Ornibactins? A new family of siderophores from Pseudomonas. Biometals 6: 93-100.

999 Thomas, M.S. (2007) Iron acquisition mechanisms of the Burkholderia cepacia complex. BioMetals 20: 431-452.

1001 Uroz, S., Calvaruso, C., Turpault, M.-P., and Frey-Klett, P. (2009a) Mineral weathering by bacteria: ecology, actors and mechanisms. Trends Microbiol 17: $378-387$.

1004 Uroz, S., Calvaruso, C., Turpault, M.-P., Pierrat, J.C., Mustin, C., and Frey-Klett, P. (2007) Effect of the mycorrhizosphere on the genotypic and metabolic diversity of the bacterial communities Involved in mineral weathering in a forest soil. App/ Environ Microbio/73: 3019-3027.

1008 Uroz, S., Calvaruso, C., Turpault, M.-P., Sarniguet, A., de Boer, W., Leveau, J.H.J., 

2186.

1012 Uroz, S., Kelly, L.C., Turpault, M.-P., Lepleux, C., and Frey-Klett, P. (2015) The 1013 mineralosphere concept: mineralogical control of the distribution and function of mineral-associated bacterial communities. Trends Microbio/23: 751-762.

1015 Uroz, S., Oger, P., Morin, E., and Frey-Klett, P. (2012) Distinct ectomycorrhizospheres share similar bacterial communities as revealed by pyrosequencing-based analysis of 16S rRNA genes. Appl Environ Microbiol 78: 3020-3024.

1019 Uroz, S., Tech, J.J., Sawaya, N.A., Frey-Klett, P., and Leveau, J.H.J. (2014) 1020 Structure and function of bacterial communities in ageing soils: Insights from the Mendocino ecological staircase. Soil Biol Biochem 69: 265-274.

1022 Vallenet, D. (2006) MaGe: a microbial genome annotation system supported by 1023 synteny results. Nucleic Acids Res 34: 53-65.

1024 Vargas-Straube, M.J., Cámara, B., Tello, M., Montero-Silva, F., Cárdenas, F., and 

PLOS ONE 11: e0151273.

1028 Yang, H.M., Chaowagul, W., and Sokol, P.A. (1991) Siderophore production by 1029 Pseudomonas pseudomallei. Infect Immun 59: 776-780. 
1034 Table 1: List of bacterial strains, constructions and primers used in this study 


\section{Strains}

\section{Collimonas pratensis}

Strain PMB3(1)

Wild type strain

L 
$\mathrm{DH} 5 \alpha$

sup E44, $\Delta$ lacU169, (ФlacZ $\Delta M 15)$, recA1, endA1, hsdR17, thi-1, gyrA96, L

relA1 
I

I

e 
Plasmids

pUC1318

$A m p^{R}, \mathrm{Gm}^{\mathrm{R}}$

L

b

C

I

I 
pGEM-T Easy

$A m p^{R}$ 
y 
Primers

For-NRPS

Rev-NRPS
5'-GCCCATCCAATACGCCGATTAC-3'

5'-GGTCGGACCGTAGTGATTGATG-3'
$\operatorname{Tm}\left({ }^{\circ} \mathrm{C}\right)$

54

This study

This study

1035

1036 


\section{Table 2: Description of the NRPS region involved in siderophore production by Collimonas pratensis strain PMB3(1)}

1038 Each gene of the region of interest is presented with its NCBI accession number. Both MaGe and NCBI annotations are displayed.

1039 The putative function of each gene is indicated by a color code (regulation (purple); siderophore biogenesis (green) including NRPS

1040 (red); transport and utilization of the siderophore (blue); unknown function (grey)).

1041

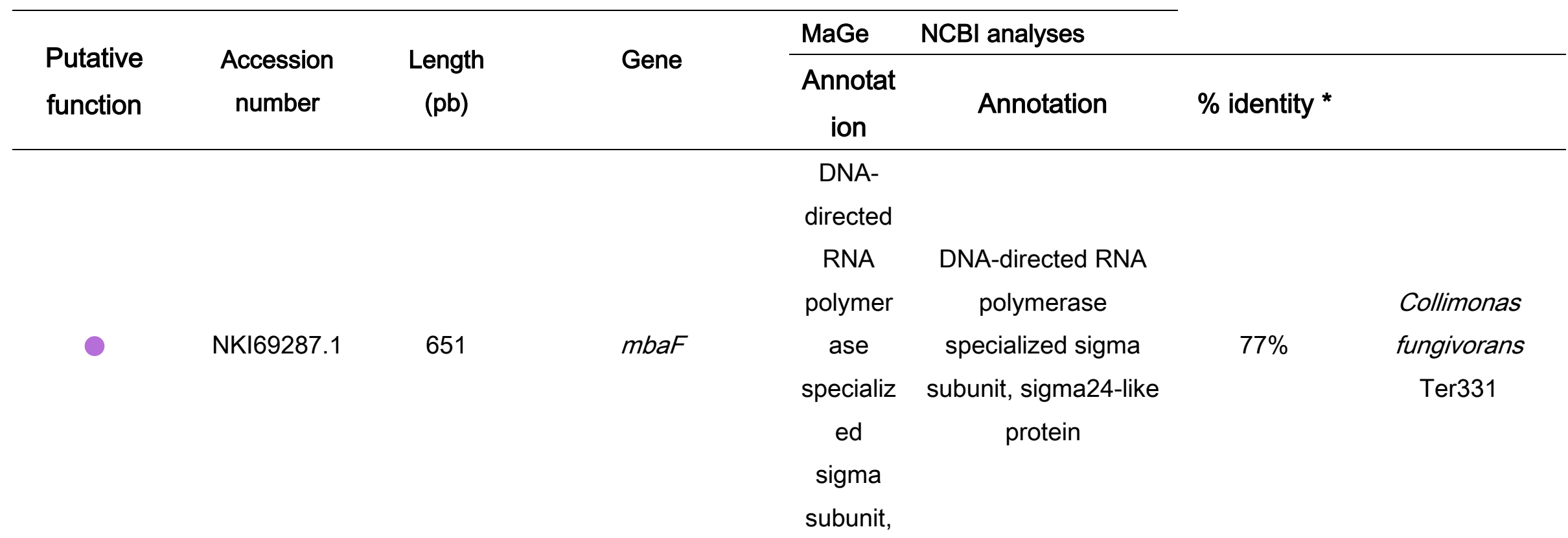




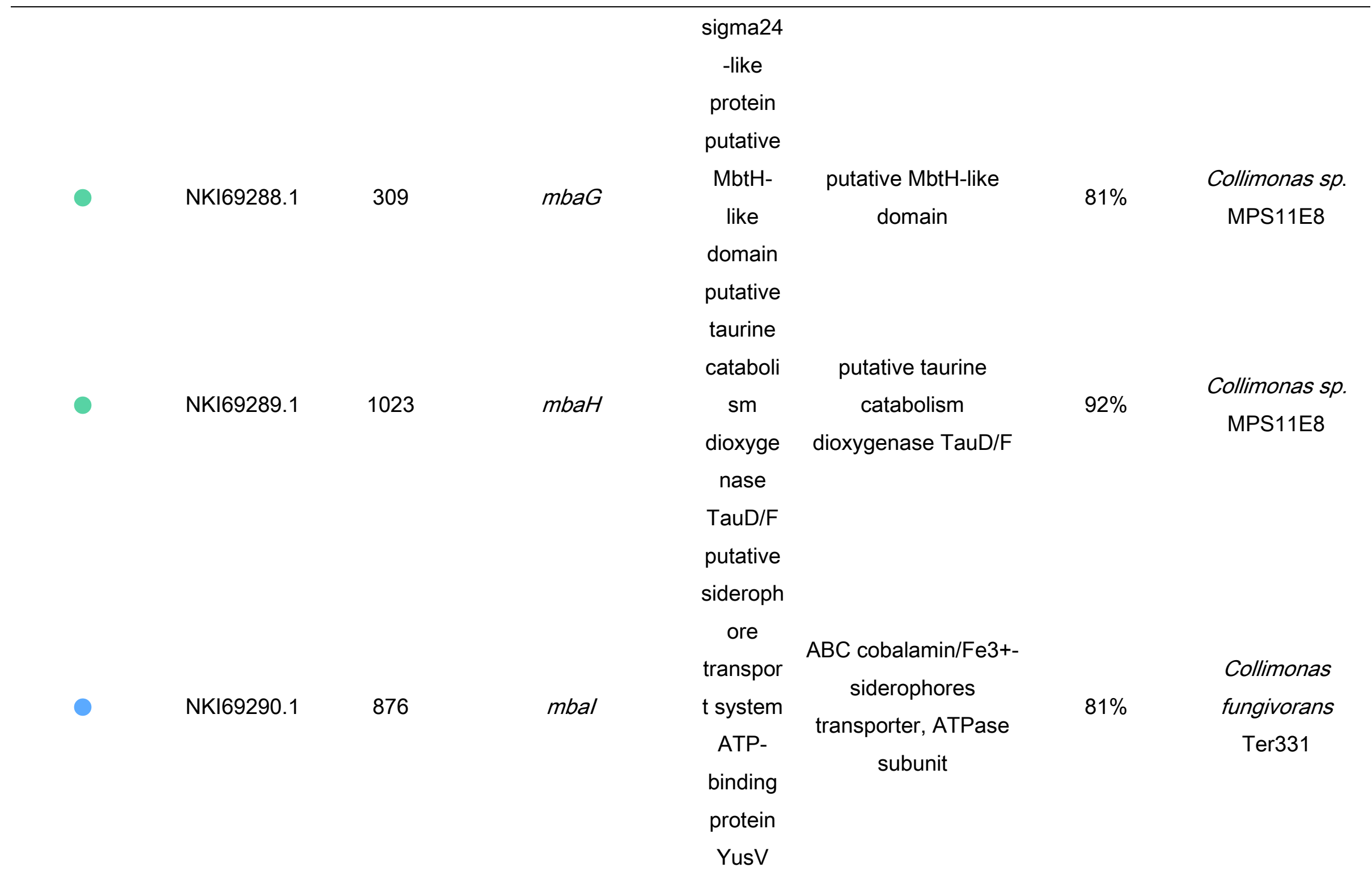




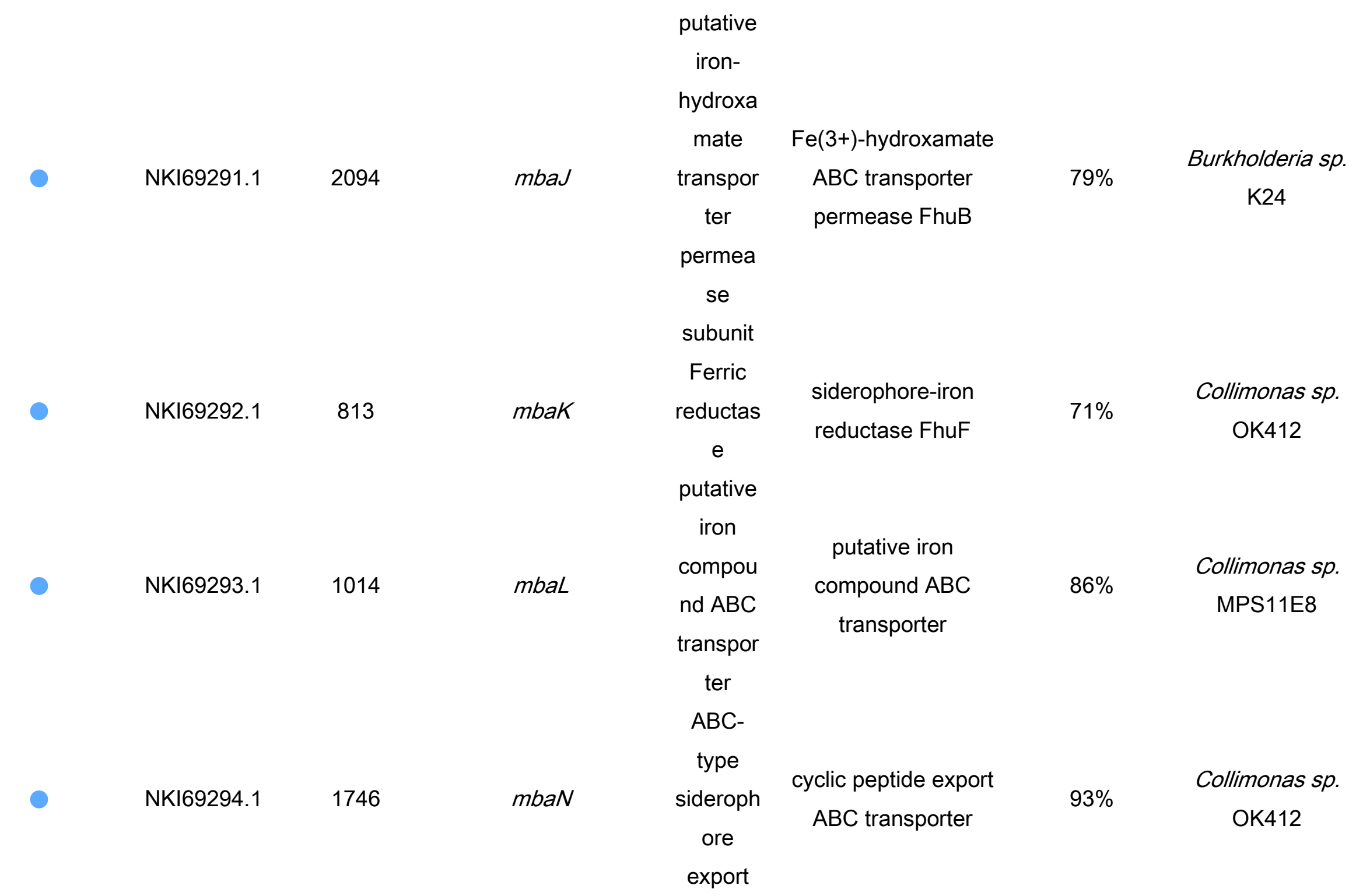




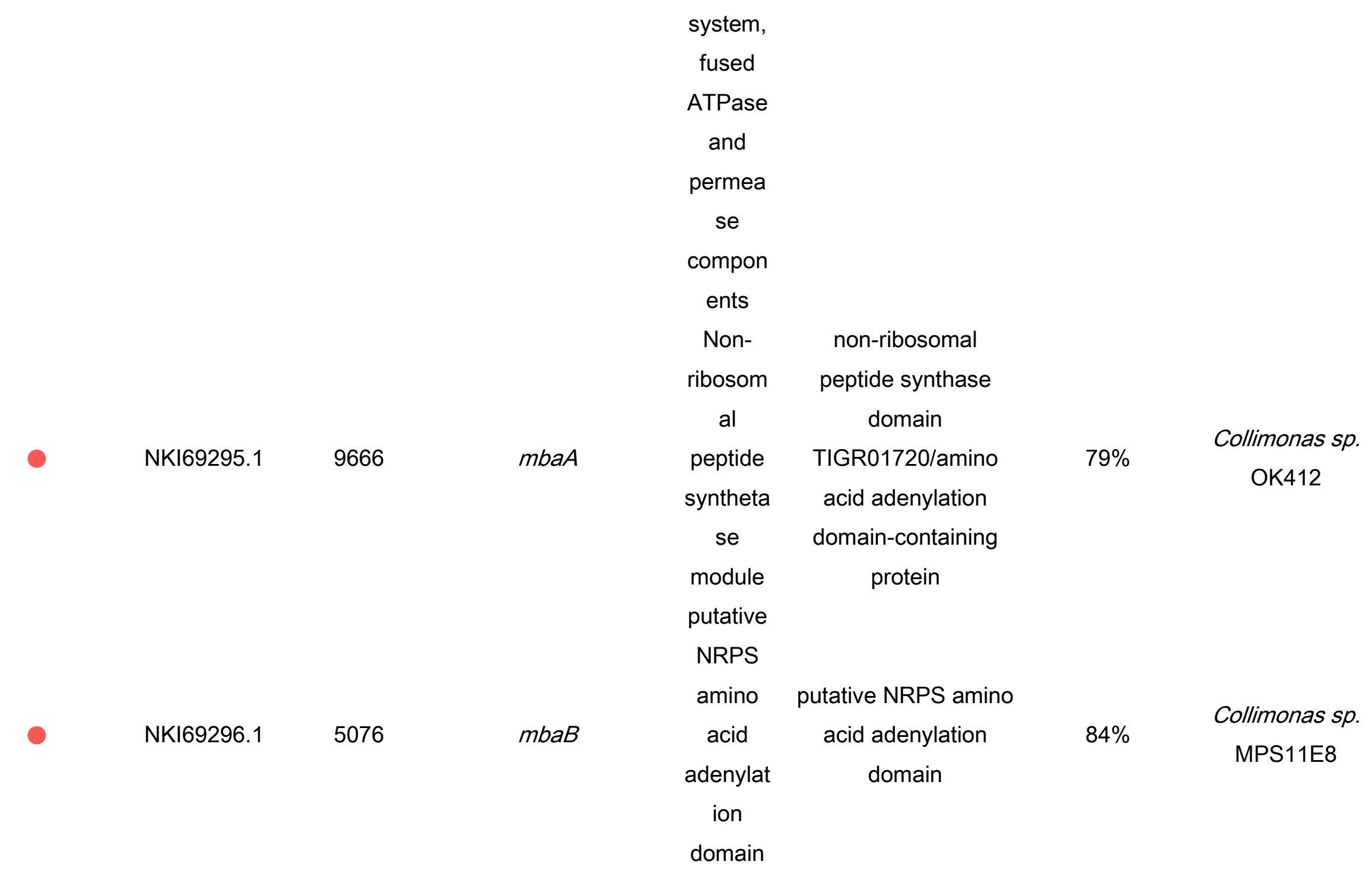


NKI69297.

1374

2220

NKI69298.1

822

NKI69300.1

2358

NKI69299.1 mbac

$m b a D$

$m b a E$
L-

ornithine

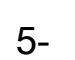

putative L-ornithin-5-

monooxygenase

$96 \%$

Collimonas $s p$.

monoox

ygenase

putative

TonB-

depend

putative TonB-

ent

dependent

siderophore receptor

partial

sideroph

ore

receptor

$\mathrm{N}(5)$ -

hydroxy

ornithine $\mathrm{N}(5)$-hydroxyornithine

formylt

transformylase, PvdF-

type

$89 \%$

ansferas

e, PvdF-

type

Isoquino

xanthine

line 1- dehydrogenase family

protein

$95 \%$

iorB

oxidored

molybdopterin-binding
Collimonas $s p$.

MPS11E8

Collimonas $s p$.

MPS11E8

Collimonas $s p$.

OK412

$\mathrm{PA}-\mathrm{H} 2$ 


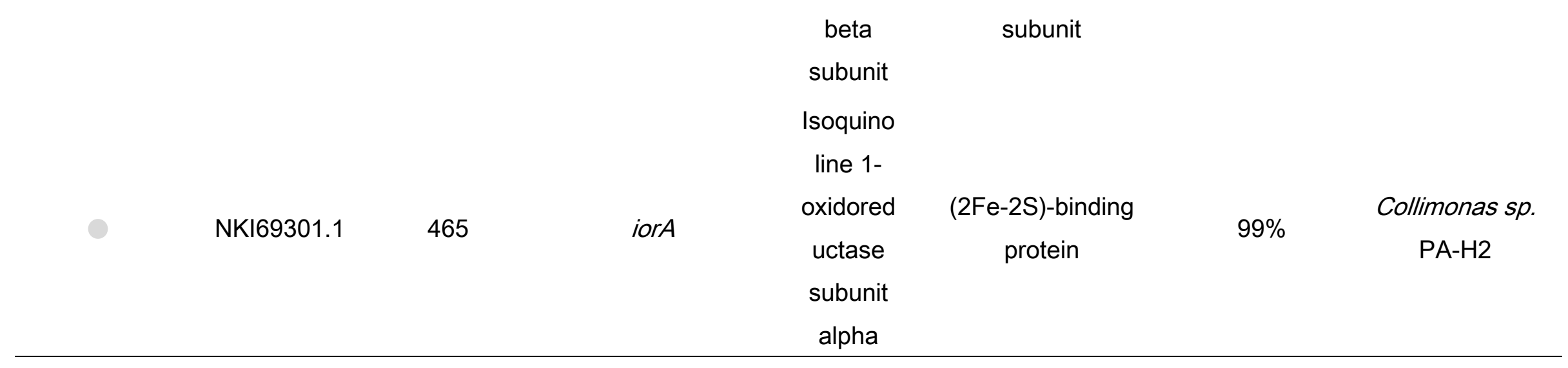


1042 Figure 1: Impact of increasing concentrations of iron on chelating and growth abilities

1043 Siderophore activity. (A) The Collimonas pratensis strain PMB3(1) WT (top) and its

$1044 \Delta$ NRPS mutant (bottom) were incubated for 3 days at $25^{\circ} \mathrm{C}$ on CAS solid medium.

1045 The presence of a siderophore activity is determined by the production of yellow halo

1046 around the colony. (B) To determine the concentration of iron inhibiting siderophore

1047 production, liquid cultures of WT (red) and $\triangle$ NRPS (blue) were performed with

1048 different concentrations of iron (i.e. $0.5 ; 0.6 ; 0.7 ; 0.8 ; 0.9 ; 1.0 \mathrm{mg} / \mathrm{l}$ ). Pure water

1049 (black) was used as a negative control. After 3 days, the absorbance at $655 \mathrm{~nm}$ was

1050 measured. The absorbance measured at $655 \mathrm{~nm}$ detects blue colour, yellow colour

1051 (corresponding to siderophore activity) will result of a decrease of the absorbance

1052 measured.

1053 Growth assay. (C) The growth of the WT strain (red) and $\triangle$ NRPS mutant (blue) was

1054 monitored in presence (full circle) or absence (empty circle) of EDTA (10 mM) and

1055 with iron $(1 \mathrm{mg} / \mathrm{l})$ in $A B$ medium ( $2 \mathrm{~g} / \mathrm{l}$ mannitol). The growth was performed under

1056 orbital shaking at $25^{\circ} \mathrm{C}$ for $90 \mathrm{~h}$. The absorbance was measured at $600 \mathrm{~nm}$ every 3

1057 hours. Each dot is the mean of independent triplicates. 
1059 Figure 2: Organization of the genomic region involved in the biosynthesis of 1060 siderophore in Collimonas pratensis strain PMB3(1)

1061 Each wide arrow represents one gene and the size is according to the gene length.

1062 Gene annotation is shown under each gene and the complete description is provided

1063 in Table 2. Fine grey arrows represent promoters. Thin black arrows represent $m b a F-$

1064 dependent promoters with specific -35 and -10 regions (TAAA/n(17)/CGTC)

1065 (Thomas, 2007). The purple gene encodes a putative sigma regulation factor. Green

1066 genes are responsible for the siderophore biosynthesis and the two NRPS genes are

1067 specifically represented in red. The blue genes are involved in the siderophore

1068 utilisation and transport. The two white genes have unknown function in siderophore

1069 biosynthesis or utilisation, and represent potential accessory genes involved in

1070 siderophore production. The little red arrow in the $m b a A$ gene indicates the Smal

1071 restriction site used for the insertion of the gentamycin resistance cassette to create

1072 the $\triangle$ NRPS mutant.

1073

1074 Figure 3: HPLC analysis and determination of the siderophore activity in the fractions

1075 collected 
1076 (A) HPLC analysis and UV detection. Products analysis of the pre-purified

1077 supernatant of the WT strain (red), $\triangle$ NRPS mutant (blue) and non-inoculated medium

1078 (black) were monitored at $210 \mathrm{~nm}$ after HPLC separation using a Milli-Q water /

1079 acetonitrile gradient for $40 \mathrm{~min}$ at $1 \mathrm{ml} / \mathrm{min}$. A major peak at $1.2 \mathrm{~min}$ retention time

1080 and two smaller peaks at 2.2 and 3.8 min retention time were detected in the

1081 supernatant of the WT strain. These three peaks are highlighted by red arrows. (B)

1082 Overlay of the HPLC and CAS analyses. To determine the presence of a siderophore

1083 activity, fractions were collected during the HPLC run and tested using the liquid CAS

1084 assay (activity level on the right axis). The HPLC spectra of the WT strain (red line;

1085 left axis), the mutant $\triangle$ NRPS (blue line; left axis) and the medium (black line; left

1086 axis) were overlaid with the siderophore activity measured by the CAS test on the

1087 fractions for the WT strain (red dotted line; right axis), the mutant $\triangle$ NRPS (blue

1088 dotted line; right axis) and the medium (black dotted line; right axis).

1089

1090 Figure 4: Chemical characterization of siderophore and ferrisiderophore

$1091 \mathrm{ESI}^{+}-\mathrm{HRMS}$ analysis of the fraction collected at $1.2 \mathrm{~min}$ (after HPLC purification) from

1092 the crude extract of the WT strain was performed. (A) Without prior incubation with 87 
1093 iron, the spectrum shows a major signal at $\mathrm{m} / \mathrm{z}=623.3069$ (mono-protonated ion

$\left.1094[\mathrm{M}+\mathrm{H}]^{+}\right)$which indicates a siderophore exact mass of $M_{S}=622.3069$. (B) After prior

1095 incubation with iron, the analysis reveals a major signal at $\mathrm{m} / \mathrm{z}=676.2191$ (mono-

1096 protonated ion $[\mathrm{M}+\mathrm{H}]^{+}$) which indicates a ferrosiderophore exact mass of $\mathrm{MFS}_{\mathrm{FS}}=$

1097 675.2191. The isotopic pattern detailed in the dashed inset shows the obvious

1098 presence of an iron atom in the structure, thanks to the clear contributions of ${ }^{54} \mathrm{Fe}$

1099 and $56 \mathrm{Fe}$ (i.e., $\mathrm{m} / \mathrm{z}=674.2246$ with $5.7 \%$ ratio and $\mathrm{m} / \mathrm{z}=676.2191$ respectively). (C)

1100 MS/MS spectrum. The previously highlighted siderophore compound (MS =

1101 622.3069) was further analyzed by ESI-MS/MS to validate its structure. M/z values

1102 framed in red correspond to theoretical fragments consecutive to breaking of the

1103 peptide bonds of the expected siderophore $(\mathrm{m} / \mathrm{z}$ values framed in orange correspond

1104 to the dehydrated forms). (D) Theoretical structure and fragmentation of malleobactin

$1105 \mathrm{X}$. Each letter represents the amino acid letter code forming the malleobactin $\mathrm{X}$. The

1106 cleavage of the different peptide bonds (blue dashed lines) results in the formation of

1107 different ions containing the $\mathrm{N}$-terminus of the peptide (b) and to ions containing the

1108 C-terminus of the peptide (y). Depending on the peptide bond cleaved, different

1109 fragments can be formed and their mass is presented as $b(1,2,3,4)$ and $y(1,2,3,4)$. 88 
1110 The fragments identified experimentally (see panel C), which mass corresponds to

1111 the theoretical masses, are presented in red, the other are presented in blue. (E)

1112 Structure and composition of the malleobactin $\mathrm{X}$. The molecule represented

1113 corresponds to the reference malleobactin $\mathrm{X}$ predicted by Vargas-Straube et al.

1114 2016. The name of each amino acid is written in full under the molecule as well as its

1115 one letter abbreviation.

1116

1117 Figure 5: Phylogenetic tree based on the NRPS MbaA protein

1118 The conservation of the protein MbaA among Collimonas and other related genera

1119 (Paraburkholderia, Burkholderia, Caballeronia, Herbaspirillum) is presented on this

1120 phylogenetic tree. A set of 52 NRPS protein sequences was analyzed and is detailed

1121 in the Table S1. Our model strain Collimonas pratensis PMB3(1) is in bold. The

1122 distribution and relatedness to known NRPS types is indicated by a color code:

1123 ornibactin (i.e. Orbl-like protein: green circles), malleobactin (i.e. MbaA-like protein;

1124 red circles) or serobactin (purple circles). The MbaA homologues for which

1125 production of such siderophore was experimentally demonstrated or only predicted

1126 are presented with full circles and empty circles, respectively. The production of 89 
1127 ornibactin have been experimentally demonstrated for 3 strains included in the

1128 analysis (full green circle; (Meyer et al., 1995; Darling et al., 1998; Deng et al., 2017).

1129 Malleobactin was identified in three strains included in the analysis (full red circle;

1130 (Alice et al., 2006; Franke et al., 2013; Vargas-Straube et al., 2016). The other proteins

1131 considered in this analysis had only predicted function (empty circle; (Holden et al.,

1132 2009; Schwager et al., 2013; Song et al., 2015; Esmaeel et al., 2016, 2018). The

1133 siderophores predicted as ornibactin and discussed in this study are represented by

1134 green dotted circles. Letters inside circles correspond to the cited reference (in the

1135 figure) in which the prediction or demonstration was/were done.

1137 Figure 6: Determination of the weathering ability of the WT Collimonas pratensis

1138 strain PMB3(1) and its $\triangle$ NRPS mutant

1139 The hematite weathering potential of strain PMB3(1) and its $\triangle$ NRPS mutant was

1140 evaluated by the measure of iron released from hematite in ABm medium devoid of

1141 iron, after 7 days of incubation at $25^{\circ} \mathrm{C}$ under agitation (200 rpm). Non-inoculated

1142 media with and without hematite (termed Hematite and Medium respectively) were

1143 used as control. (A) Quantity of iron released in solution. The iron released from 90 
1144 hematite was measured by ferrospectral determination (optical density measured at

$1145595 \mathrm{~nm})$. A standard calibration of iron $\left(\mathrm{FeCl}_{3}\right)$ was used to calculate the iron

1146 concentration of the different samples tested (WT, $\triangle$ NRPS, hematite alone or

1147 Medium alone). Samples with the same letter indicate no significant difference

$1148(\mathrm{P}<0.05) .(\mathrm{B}) \mathrm{pH}$ of the supernatant. The acidification of the medium was measured

1149 by absorbance at $595 \mathrm{~nm}$ using the bromocresol green method. (C) Siderophore

1150 activity. The siderophore activity of the different samples was determined using the

1151 CAS method. The yellow colour indicates a siderophore activity. For each measure,

1152 data are presented under the corresponding pictures and are the mean of

1153 independent triplicates, the standard deviation is indicated in italic. 